\title{
Congruence classes of points in quaternionic hyperbolic space
}

\author{
Wensheng Cao \\ School of Mathematics and Computational Science, \\ Wuyi University, Jiangmen, \\ Guangdong 529020, P.R. China \\ e-mail: wenscao@aliyun.com
}

\begin{abstract}
An important problem in quaternionic hyperbolic geometry is to classify ordered $m$-tuples of pairwise distinct points in the closure of quaternionic hyperbolic n-space, $\overline{\mathbf{H}_{\mathbb{H}}^{n}}$, up to congruence in the holomorphic isometry group $\operatorname{PSp}(n, 1)$ of $\mathbf{H}_{\mathbb{H}}^{n}$. In this paper we concentrate on two cases: $m=3$ in $\overline{\mathbf{H}_{\mathbb{H}}^{n}}$ and $m=4$ on $\partial \mathbf{H}_{\mathbb{H}}^{n}$ for $n \geq 2$. New geometric invariants and several distance formulas in quaternionic hyperbolic geometry are introduced and studied for this problem. The congruence classes are completely described by quaternionic Cartan's angular invariants and the distances between some geometric objects for the first case. The moduli space is constructed for the second case.
\end{abstract}

Mathematics Subject Classifications (2000) 57M50, 53C17, 32M15.

Keywords Quaternionic cross-ratio; Quaternionic Cartan's angular invariant; Gram matrix; Congruence class; Moduli space.

\section{Introduction}

Let $\mathbb{F}$ denote the real numbers $\mathbb{R}$, the complex numbers $\mathbb{C}$ or the quaternions $\mathbb{H}$. Let $\mathbf{H}_{\mathbb{F}}^{n}$ denote the $n$ dimensional hyperbolic space over $\mathbb{F}$ with the boundary $\partial \mathbf{H}_{\mathbb{F}}^{n}$. Let $\langle$,$\rangle be the Hermitian product in \mathbb{F}^{n+1}$ of signature $(n, 1)$. For $\mathbb{F}=\mathbb{C}$ and $\mathbb{H}$, the linear groups which act as the isometries in $\mathbf{H}_{\mathbb{F}}^{n}$, are denoted by $\mathrm{PU}(n, 1)$ and $\operatorname{PSp}(n, 1)$ respectively. An important problem in hyperbolic geometry is to classify ordered $m$-tuples of pairwise distinct points in the closure of hyperbolic n-space $\overline{\mathbf{H}_{\mathbb{F}}^{n}}$ up to congruence in the holomorphic isometry group of $\mathbf{H}_{\mathbb{F}}^{n}$. This problem is trivial for $m=1,2$. To deal with the cases of $m \geq 3$, one need to develop some geometric invariants or geometric tools.

The cross-ratio of a quadruple of points in $\partial \mathbf{H}_{\mathbb{R}}^{n}$ was defined by Cao and Waterman [5], which coincides with the classical cross-ratio of the complex plane when $n=3$ and the quaternionic cross-ratio of a quadruple of quaternions [3] when $n=5$. These classical cross ratios are useful tools in real hyperbolic geometry.

Let $\mathfrak{p}=\left(p_{1}, p_{2}, p_{3}, p_{4}\right)$ be an ordered quadruple of pairwise distinct points in $\partial \mathbf{H}_{\mathbb{C}}^{n}$. The classical cross-ratio was generalized to $\partial \mathbf{H}_{\mathbb{C}}^{n}$ by Korányi and Reimann [16] as the following complex number:

$$
\mathbb{X}(\mathfrak{p})=\mathbb{X}\left(p_{1}, p_{2}, p_{3}, p_{4}\right)=\frac{\left\langle\mathbf{p}_{3}, \mathbf{p}_{1}\right\rangle\left\langle\mathbf{p}_{4}, \mathbf{p}_{2}\right\rangle}{\left\langle\mathbf{p}_{4}, \mathbf{p}_{1}\right\rangle\left\langle\mathbf{p}_{3}, \mathbf{p}_{2}\right\rangle}
$$

where $\mathbf{p}_{i} \in \mathbb{C}^{n, 1}$ are null lifts of $p_{i}$. This complex cross-ratio is closely related to Cartan's angular invariant and other geometric invariants. The Cartan's angular invariant [7, 13] is an angle associated 
to a triple $\mathfrak{p}=\left(p_{1}, p_{2}, p_{3}\right)$ of points in $\partial \mathbf{H}_{\mathbb{C}}^{n}$. Such an angle $\mathbb{A}(\mathfrak{p})$ is defined to be the following argument:

$$
\mathbb{A}(\mathfrak{p})=\arg \left(-\left\langle\mathbf{p}_{1}, \mathbf{p}_{2}, \mathbf{p}_{3}\right\rangle\right) \in[-\pi / 2, \pi / 2],
$$

where $\mathbf{p}_{i}$ are the null lifts of $p_{i}$, and

$$
\left\langle\mathbf{p}_{1}, \mathbf{p}_{2}, \mathbf{p}_{3}\right\rangle=\left\langle\mathbf{p}_{1}, \mathbf{p}_{2}\right\rangle\left\langle\mathbf{p}_{2}, \mathbf{p}_{3}\right\rangle\left\langle\mathbf{p}_{3}, \mathbf{p}_{1}\right\rangle .
$$

It is a shape invariant, originally used in detecting whether the corresponding triple lies on a chain or on an $\mathbb{R}$-circle.

The Cartan's angular invariant $\mathbb{A}(\mathfrak{p})$ and some distance formulas between some geometric objects are the exact geometric invariant and tools to study congruence classes of triples $\mathfrak{p}=\left(p_{1}, p_{2}, p_{3}\right) \in\left(\overline{\mathbf{H}_{\mathbb{C}}^{n}}\right)^{3}$. The Cartan's angular invariant $\mathbb{A}(\mathfrak{p})$ of a tripe $\mathfrak{p}=\left(p_{1}, p_{2}, p_{3}\right) \in\left(\partial \mathbf{H}_{\mathbb{C}}^{n}\right)^{3}$ determines its congruence class in $\mathrm{PU}(n, 1)$ [7, 13. The moduli space of such triples can be described as the interval $[-\pi / 2, \pi / 2]$. If such a triple $\mathfrak{p}=\left(p_{1}, p_{2}, p_{3}\right)$ is in $\mathbf{H}_{\mathbb{C}}^{n}$, then its congruence class in $\mathrm{PU}(n, 1)$ is described by the three distance $\rho\left(p_{i}, p_{j}\right)$ and Brehm's shape invariant [4]. Here $\rho($,$) is the Bergman metric on \mathbf{H}_{\mathbb{C}}^{n}$. The distance formula from a point in $\mathbf{H}_{\mathbb{C}}^{n}$ to a complex geodesic is needed for the general congruence class problem in $\overline{\mathbf{H}_{\mathbb{C}}^{n}}$.

For $n=2$ and $m=4$ this problem was considered by Falbel, Parker and Platis [11, 12, 18, 19]. The main tool is the complex cross-ratio variety determined by three complex cros-ratios. The moduli space of ordered quadruples of pairwise distinct points in $\partial \mathbf{H}_{\mathbb{C}}^{n}$ was described by Cunha and Gusevskii [9] with the tool of Gram matrix. A Gram matrix associated to $\mathfrak{p}=\left(p_{1}, p_{2}, p_{3}, p_{4}\right)$ in $\partial \mathbf{H}_{\mathbb{H}}^{n}$ with lift $\mathbf{p}=\left(\mathbf{p}_{1}, \mathbf{p}_{2}, \mathbf{p}_{3}, \mathbf{p}_{4}\right)$ is the Hermitian matrix

$$
G=G(\mathbf{p})=\left(g_{i j}\right)=\left(\left\langle\mathbf{p}_{i}, \mathbf{p}_{j}\right\rangle\right) .
$$

Gram matrix is an important tool in complex hyperbolic geometry [9, 13, 14] because its entries $\left\langle\mathbf{p}_{i}, \mathbf{p}_{j}\right\rangle$ are base material for other geometric invariants. We can read off it almost all the geometric information concerning the relative geometric positions of $p_{i}$.

This technique is also used to construct the invariants which describe uniquely the $\mathrm{PU}(n, 1)$-congruence class of an ordered $m$-tuple of pairwise distinct points in $\partial \mathbf{H}_{\mathbb{C}}^{n}$ and describe the corresponding moduli space for any $n \geq 1$ and $m \geq 4[10$.

The main aim of this paper is concerned with the important problem mention above in quaternionic hyperbolic geometry. We concentrate ourself on two cases: $m=3$ in $\overline{\mathbf{H}_{\mathbb{H}}^{n}}$ and $m=4$ on $\partial \mathbf{H}_{\mathbb{H}}^{n}$ for $n \geq 2$. We will consider the description of congruence classes for the first case. We also will obtain a construction of the moduli space of ordered quadruples of pairwise distinct points in $\partial \mathbf{H}_{\mathbb{H}}^{n}$.

For this purpose we need to develop some geometric invariants and tools in quaternionic hyperbolic geometry. We will extend the quaternionic Cartan's angular invariant and quaternionic cross-ratio to $\overline{\mathbf{H}_{\mathbb{H}}^{n}}$. Several distance formulas between some geometric objects are obtained. We will introduce the Gram matrix in quaternionic hyperbolic geometry. We remark that some generalizations are new even in complex hyperbolic geometry and the introduced geometric invariants deserve further research in quaternionic hyperbolic geometry.

We need several notations and definitions to state our main results. Such notations and definitions rely on the following two propositions, which will be proved in Section 2 below.

Proposition 1.1. If $\mathbf{z}, \mathbf{w} \in \mathbb{H}^{n, 1}-\{0\}$ with $\langle\mathbf{z}, \mathbf{z}\rangle \leq 0$ and $\langle\mathbf{w}, \mathbf{w}\rangle \leq 0$ then either $\mathbf{w}=\mathbf{z} \lambda$ for some $\lambda \in \mathbb{H}$ or $\langle\mathbf{z}, \mathbf{w}\rangle \neq 0$.

Proposition 1.2. Let $\mathfrak{p}=\left(p_{1}, p_{2}, p_{3}\right)$ be any triple of pairwise distinct points in $\overline{\mathbf{H}_{\mathbb{H}}^{n}}$ and $\mathbf{p}_{1}, \mathbf{p}_{2}, \mathbf{p}_{3}$ be arbitrary lifts of $p_{1}, p_{2}, p_{3}$ respectively, then the number

$$
\left\langle\mathbf{p}_{1}, \mathbf{p}_{2}, \mathbf{p}_{3}\right\rangle=\left\langle\mathbf{p}_{2}, \mathbf{p}_{1}\right\rangle\left\langle\mathbf{p}_{3}, \mathbf{p}_{2}\right\rangle\left\langle\mathbf{p}_{1}, \mathbf{p}_{3}\right\rangle \in \mathbb{H},
$$


and

$$
\Re\left(\left\langle\mathbf{p}_{1}, \mathbf{p}_{2}, \mathbf{p}_{3}\right\rangle\right) \leq 0 .
$$

By Propositions 1.11.2, we can rephrase the definition of quaternionic Cartan's angular invariant by Apanasov and Kim [1] as follows.

Definition 1.1. The quaternionic Cartan's angular invariant of a triple $\mathfrak{p}=\left(p_{1}, p_{2}, p_{3}\right)$ of pairwise distinct points in $\overline{\mathbf{H}_{\mathbb{H}}^{n}}$ is the angular invariant $\mathbb{A}_{\mathbb{H}}(\mathfrak{p}), 0 \leq \mathbb{A}_{\mathbb{H}}(\mathfrak{p}) \leq \frac{\pi}{2}$, given by

$$
\mathbb{A}_{\mathbb{H}}(\mathfrak{p})=\mathbb{A}_{\mathbb{H}}\left(p_{1}, p_{2}, p_{3}\right):=\arccos \frac{\Re\left(-\left\langle\mathbf{p}_{1}, \mathbf{p}_{2}, \mathbf{p}_{3}\right\rangle\right)}{\left|\left\langle\mathbf{p}_{1}, \mathbf{p}_{2}, \mathbf{p}_{3}\right\rangle\right|}
$$

where $\mathbf{p}_{1}, \mathbf{p}_{2}, \mathbf{p}_{3}$ are lifts of $p_{1}, p_{2}, p_{3}$, respectively.

Definition 1.2. For $u, v \in \overline{\mathbf{H}_{\mathbb{H}}^{n}}$ with lifts $\mathbf{u}$ and $\mathbf{v}$, respectively, we define the quaternionic line spanned by $u, v$ as the set

$$
L_{u v}=\mathbb{P}(\{\mathbf{w}: \mathbf{w}=\mathbf{u} \lambda+\mathbf{v} \mu, \lambda, \mu \in \mathbb{H}\}) \cap \overline{\mathbf{H}_{\mathbb{H}}^{n}} .
$$

Let $\rho\left(\right.$, ) be the Bergman metric on $\mathbf{H}_{\mathbb{H}}^{n}$ and $\rho\left(L_{u v}, z\right)$ the hyperbolic distance from $z \in \mathbf{H}_{\mathbb{H}}^{n}$ to $L_{u v}$.

One of our main results is the following theorem.

Theorem 1.1. Let $\mathfrak{p}=\left(p_{1}, p_{2}, p_{3}\right)$ and $\mathfrak{q}=\left(q_{1}, q_{2}, q_{3}\right)$ be triples of pairwise distinct points in $\overline{\mathbf{H}_{\mathbb{H}}^{n}}$. Then there exists an isometry $h \in \operatorname{Sp}(n, 1)$ such that $h\left(p_{1}\right)=q_{1}, h\left(p_{2}\right)=q_{2}, h\left(p_{3}\right)=q_{3}$ if and only if one of the following conditions holds:

(i) $\mathfrak{p}, \mathfrak{q} \in \partial \mathbf{H}_{\mathbb{H}}^{n} \times \partial \mathbf{H}_{\mathbb{H}}^{n} \times \partial \mathbf{H}_{\mathbb{H}}^{n}$ and $\mathbb{A}_{\mathbb{H}}(\mathfrak{p})=\mathbb{A}_{\mathbb{H}}(\mathfrak{q})$.

(ii) $\mathfrak{p}, \mathfrak{q} \in \partial \mathbf{H}_{\mathbb{H}}^{n} \times \partial \mathbf{H}_{\mathbb{H}}^{n} \times \mathbf{H}_{\mathbb{H}}^{n}, \mathbb{A}_{\mathbb{H}}(\mathfrak{p})=\mathbb{A}_{\mathbb{H}}(\mathfrak{q})$ and $\rho\left(L_{p_{1} p_{2}}, p_{3}\right)=\rho\left(L_{q_{1} q_{2}}, q_{3}\right)$.

(iii) $\mathfrak{p}, \mathfrak{q} \in \partial \mathbf{H}_{\mathbb{H}}^{n} \times \mathbf{H}_{\mathbb{H}}^{n} \times \mathbf{H}_{\mathbb{H}}^{n}, \rho\left(L_{p_{1} p_{2}}, p_{3}\right)=\rho\left(L_{q_{1} q_{2}}, q_{3}\right), \rho\left(L_{p_{1} p_{3}}, p_{2}\right)=\rho\left(L_{q_{1} q_{3}}, q_{2}\right)$ and $\rho\left(p_{2}, p_{3}\right)=$ $\rho\left(q_{2}, q_{3}\right)$.

(iv) $\mathfrak{p}, \mathfrak{q} \in \mathbf{H}_{\mathbb{H}}^{n} \times \mathbf{H}_{\mathbb{H}}^{n} \times \mathbf{H}_{\mathbb{H}}^{n}, \mathbb{A}_{\mathbb{H}}(\mathfrak{p})=\mathbb{A}_{\mathbb{H}}(\mathfrak{q}), \rho\left(p_{1}, p_{2}\right)=\rho\left(q_{1}, q_{2}\right), \rho\left(p_{1}, p_{3}\right)=\rho\left(q_{1}, q_{3}\right)$ and $\rho\left(p_{2}, p_{3}\right)=$ $\rho\left(q_{2}, q_{3}\right)$.

For two quaternions $a=a_{0}+a_{1} \mathbf{i}+a_{2} \mathbf{j}+a_{3} \mathbf{k}$ and $b=b_{0}+b_{1} \mathbf{i}+b_{2} \mathbf{j}+b_{3} \mathbf{k}$, where $a_{i}, b_{i} \in \mathbb{R}$. We define the following two functions:

$$
\nu: \mathbb{H} \rightarrow \mathbb{H}, \sigma: \mathbb{H} \times \mathbb{H} \rightarrow \mathbb{C}
$$

as

$$
\nu(a)= \begin{cases}\frac{\left(\sqrt{a_{1}^{2}+a_{2}^{2}+a_{3}^{2}}+a_{1}\right)-a_{3} \mathbf{j}+a_{2} \mathbf{k}}{\sqrt{2\left(a_{1}^{2}+a_{2}^{2}+a_{3}^{2}\right)+2 a_{1} \sqrt{a_{1}^{2}+a_{2}^{2}+a_{3}^{2}}},}, & \text { provided } a_{2}^{2}+a_{3}^{2} \neq 0 \\ \mathbf{j}, & \text { provided } a_{1}<0 \\ 1, & \text { otherwise }\end{cases}
$$

and

$$
\sigma(a, b)= \begin{cases}\sqrt{\frac{a_{2}+a_{3} \mathbf{i}}{\sqrt{a_{2}^{2}+a_{3}^{2}}}}, & \text { provided } a_{2}^{2}+a_{3}^{2} \neq 0 ; \\ \sqrt{\frac{b_{2}+b_{3} \mathbf{i}}{\sqrt{b_{2}^{2}+b_{3}^{2}}}}, & \text { provided } b_{2}^{2}+b_{3}^{2} \neq 0 \\ 1, & \text { otherwise. }\end{cases}
$$


We mention that $|\nu(a)|=|\sigma(a, b)|=1$ and $\nu(a)$ and $\sigma(a, b)$ can be viewed as orthogonal rotations such that

$$
\nu(a)^{-1} a \nu(a)=a_{0}+\sqrt{a_{1}^{2}+a_{2}^{2}+a_{3}^{2}} \mathbf{i}
$$

and

$$
\sigma(a, b)^{-1} a \sigma(a, b)=a_{0}+a_{1} \mathbf{i}+\sqrt{a_{2}^{2}+a_{3}^{2}} \mathbf{j}, \text { provided } a_{2}^{2}+a_{3}^{2} \neq 0
$$

or

$$
\sigma(a, b)^{-1} b \sigma(a, b)=b_{0}+b_{1} \mathbf{i}+\sqrt{b_{2}^{2}+b_{3}^{2}} \mathbf{j}, \text { provided } a_{2}^{2}+a_{3}^{2}=0, b_{2}^{2}+b_{3}^{2} \neq 0 .
$$

Let $\mathcal{M}(n)$ be the configuration space of quadruples of pairwise distinct points in $\partial \mathbf{H}_{\mathbb{H}}^{n}$, that is, the quotient of the set of quadruples of pairwise distinct points in $\partial \mathbf{H}_{\mathbb{H}}^{n}$ with respect to the diagonal action of $\operatorname{PSp}(n, 1)$ equipped with the quotient topology.

Let $m(\mathfrak{p}) \in \mathcal{M}(n)$ be the point represented by $\mathfrak{p}=\left(p_{1}, p_{2}, p_{3}, p_{4}\right)$. We define the map

$$
\tau: \mathcal{M}(n) \rightarrow \mathbb{C}^{3} \times \mathbb{R} \times \mathbb{R}
$$

by the formula

$$
\tau: m(\mathfrak{p}) \longmapsto\left(c_{1}, c_{2}, c_{3}, t ; \mathbb{A}\right),
$$

where $\left(c_{1}, c_{2}, c_{3}, t ; \mathbb{A}\right)$ are determined by the following three steps:

Step 1 : Determine $\lambda_{i}, i=1,2,3,4$.

Let $\mathbf{p}=\left(\mathbf{p}_{1}, \mathbf{p}_{2}, \mathbf{p}_{3}, \mathbf{p}_{4}\right)$ be an arbitrary lift of $\mathfrak{p}$. It follows from Proposition 1.1 that $\left\langle\mathbf{p}_{i}, \mathbf{p}_{j}\right\rangle \neq 0$ for $i \neq j$.

Set

$$
\lambda_{2}=\left\langle\mathbf{p}_{2}, \mathbf{p}_{1}\right\rangle^{-1}, \lambda_{3}=\left\langle\mathbf{p}_{3}, \mathbf{p}_{2}\right\rangle^{-1}\left\langle\mathbf{p}_{1}, \mathbf{p}_{2}\right\rangle, \lambda_{4}=\left\langle\mathbf{p}_{4}, \mathbf{p}_{3}\right\rangle^{-1}\left\langle\mathbf{p}_{2}, \mathbf{p}_{3}\right\rangle\left\langle\mathbf{p}_{2}, \mathbf{p}_{1}\right\rangle^{-1}
$$

and

$$
\lambda_{1}=\frac{\nu\left(\left\langle\mathbf{p}_{1}, \mathbf{p}_{3} \lambda_{3}\right\rangle\right)}{\sqrt{\left|\left\langle\mathbf{p}_{1}, \mathbf{p}_{3} \lambda_{3}\right\rangle\right|}}=\frac{\nu\left(\left\langle\mathbf{p}_{2}, \mathbf{p}_{1}\right\rangle\left\langle\mathbf{p}_{2}, \mathbf{p}_{3}\right\rangle^{-1}\left\langle\mathbf{p}_{1}, \mathbf{p}_{3}\right\rangle\right)}{\sqrt{\left|\left\langle\mathbf{p}_{2}, \mathbf{p}_{1}\right\rangle\left\langle\mathbf{p}_{2}, \mathbf{p}_{3}\right\rangle^{-1}\left\langle\mathbf{p}_{1}, \mathbf{p}_{3}\right\rangle\right|}} .
$$

Step 2: Determine $\mu$.

Set

$$
\mu=\sigma\left(\left\langle\mathbf{p}_{1} \lambda_{1}, \mathbf{p}_{4} \lambda_{4}{\overline{\lambda_{1}}}^{-1}\right\rangle,\left\langle\mathbf{p}_{2} \lambda_{2}{\overline{\lambda_{1}}}^{-1}, \mathbf{p}_{4} \lambda_{4}{\overline{\lambda_{1}}}^{-1}\right\rangle\right) .
$$

Step 3: Determine $\left(c_{1}, c_{2}, c_{3}, t ; \mathbb{A}\right)$.

Set

$$
c_{1}+t \mathbf{j}=\bar{\mu}\left\langle\mathbf{p}_{1} \lambda_{1}, \mathbf{p}_{4} \lambda_{4}{\overline{\lambda_{1}}}^{-1}\right\rangle \mu, c_{2}+c_{3} \mathbf{j}=\bar{\mu}\left\langle\mathbf{p}_{2} \lambda_{2}{\overline{\lambda_{1}}}^{-1}, \mathbf{p}_{4} \lambda_{4}{\overline{\lambda_{1}}}^{-1}\right\rangle \mu .
$$

We will show in Section 5 that the parameters $\left(c_{1}, c_{2}, c_{3}, t ; \mathbb{A}\right)$ are independent of lifts $\mathbf{p}_{i}, i=1,2,3,4$ and are determined only by $p_{i}, i=1,2,3,4$.

Definition 1.3. Let $\mathbb{M}(2)$ be the set of points $\left(c_{1}, c_{2}, c_{3}, t ; \mathbb{A}\right)$ in $\mathbb{C}^{3} \times \mathbb{R} \times \mathbb{R}$ defined by

$$
D(G)=1+\left|c_{1}\right|^{2}+\left|c_{2}\right|^{2}+\left|c_{3}\right|^{2}+t^{2}-2 \Re\left(c_{1}\right)+2 \Re\left(c_{2} e^{-\mathbf{i} \mathbb{A}}\right)+2 \Re\left(\left(\overline{c_{1}} c_{2}+t \overline{c_{3}}\right) e^{\mathbf{i} \mathbb{A}}\right)=0
$$

subject to the following restrictions:

$$
\mathbb{A} \in[0, \pi / 2], \quad \Re\left(c_{1} \overline{c_{2}}\right)+t \Re\left(c_{3}\right) \leq 0, \Re\left(c_{2}\right) \leq 0, \quad t \geq 0, \quad\left|c_{1}\right|^{2}+t^{2} \neq 0, \quad\left|c_{2}\right|^{2}+\left|c_{3}\right|^{2} \neq 0 .
$$

Let $\mathbb{M}(n)(n>2)$ be the set of points $\left(c_{1}, c_{2}, c_{3}, t ; \mathbb{A}\right)$ in $\mathbb{C}^{3} \times \mathbb{R} \times \mathbb{R}$ defined by

$$
D(G)=1+\left|c_{1}\right|^{2}+\left|c_{2}\right|^{2}+\left|c_{3}\right|^{2}+t^{2}-2 \Re\left(c_{1}\right)+2 \Re\left(c_{2} e^{-\mathbf{i} \mathbb{A}}\right)+2 \Re\left(\left(\overline{c_{1}} c_{2}+t \overline{c_{3}}\right) e^{\mathbf{i} \mathbb{A}}\right) \leq 0
$$

subject to the following restrictions:

$$
\mathbb{A} \in[0, \pi / 2], \quad \Re\left(c_{1} \overline{c_{2}}\right)+t \Re\left(c_{3}\right) \leq 0, \Re\left(c_{2}\right) \leq 0, \quad t \geq 0, \quad\left|c_{1}\right|^{2}+t^{2} \neq 0, \quad\left|c_{2}\right|^{2}+\left|c_{3}\right|^{2} \neq 0 .
$$


We call $\mathbb{M}(n)$ the moduli space for $\mathcal{M}(n)$. $\mathbb{M}(n)$ is equipped with the topology induced from $\mathbb{C}^{3} \times \mathbb{R} \times \mathbb{R}$.

The other main result in this paper is the following theorem.

Theorem 1.2. The configuration space $\mathcal{M}(n)$ is homeomorphic to $\mathbb{M}(n)$.

The paper is organized as follows. Section 2 contains some basic facts in quaternionic hyperbolic geometry and the proof of Propositions 1.1, 1.2. Section 3 is devoted to developing some geometric invariants and geometric tools in quaternionic hyperbolic geometry. These are quaternionic cross-ratio, quaternionic Cartan's angular invariant and some distance formulas. Sections 4 contains the proof of Theorem 1.1. In Section 5, we introduce the Gram matrix in quaternionic hyperbolic geometry. Besides the proof of Theorem 1.2, we also obtain a theorem (Theorem 5.2) about the congruence classes of quadruples of pairwise distinct points on $\partial \mathbf{H}_{\mathbb{H}}^{n}$.

\section{Preliminaries}

We briefly recall some necessary material on quaternionic hyperbolic geometry here and we refer to [1, 8, 15] for further details.

We recall that a quaternion is of the form $a=a_{0}+a_{1} \mathbf{i}+a_{2} \mathbf{j}+a_{3} \mathbf{k} \in \mathbb{H}$ where $a_{i} \in \mathbb{R}$ and $\mathbf{i}^{2}=\mathbf{j}^{2}=$ $\mathbf{k}^{2}=\mathbf{i j k}=-1$. Let $\bar{a}=a_{0}-a_{1} \mathbf{i}-a_{2} \mathbf{j}-a_{3} \mathbf{k}$ and $|a|=\sqrt{\bar{a} a}=\sqrt{a_{0}^{2}+a_{1}^{2}+a_{2}^{2}+a_{3}^{2}}$ be the conjugate and modulus of $a$, respectively. We define $\Re(a)=(a+\bar{a}) / 2$ and $\Im(a)=(a-\bar{a}) / 2$. For $a, b \in \mathbb{H}$, we have

$$
\Re(a b)=\Re(b a)=\Re(\bar{a} \bar{b})=\Re(\bar{b} \bar{a}) .
$$

Two quaternions $a$ and $b$ are similar, denoted by $a \sim b$, if there exists nonzero $\lambda \in \mathbb{H}$ such that $b=\lambda a \lambda^{-1}$. We mention that $a \sim b$ if and only if $\Re(a)=\Re(b)$ and $|a|=|b|$. Let

$$
\mathbb{S}=\left\{\nu=\nu_{1} \mathbf{i}+\nu_{2} \mathbf{j}+\nu_{3} \mathbf{k}: \nu_{1}^{2}+\nu_{2}^{2}+\nu_{3}^{2}=1, v_{i} \in \mathbb{R}\right\}
$$

Every unit quaternion $\nu$ can be written as

$$
\nu=\exp (\theta \mathbf{I}):=\cos \theta+\mathbf{I} \sin \theta=\cos (-\theta)+(-\mathbf{I}) \sin (-\theta) \quad \text { for some } \theta \in[0, \pi] \text { and } \mathbf{I} \in \mathbb{S} .
$$

It is useful to view $\mathbb{H}$ as $\mathbb{H}=\mathbb{C} \oplus \mathbb{C} \mathbf{j}$. Therefore any quaternion $a=a_{0}+a_{1} \mathbf{i}+a_{2} \mathbf{j}+a_{3} \mathbf{k}$ can be uniquely expressed as

$$
a=\left(a_{0}+a_{1} \mathbf{i}\right)+\left(a_{2}+a_{3} \mathbf{i}\right) \mathbf{j}=c_{1}+c_{2} \mathbf{j}=c_{1}+\mathbf{j} \overline{c_{2}} .
$$

Let $\mathbb{H}^{n, 1}$ be the vector space with the Hermitian form of signature $(n, 1)$ given by

$$
\langle\mathbf{z}, \mathbf{w}\rangle=\mathbf{w}^{*} J \mathbf{z}=\overline{w_{1}} z_{n+1}+\overline{w_{2}} z_{2}+\cdots+\overline{w_{n}} z_{n}+\overline{w_{n+1}} z_{1}
$$

with matrix

$$
J=\left(\begin{array}{ccc}
0 & 0 & 1 \\
0 & I_{n-1} & 0 \\
1 & 0 & 0
\end{array}\right)
$$

We define

$$
\operatorname{Sp}(n, 1)=\left\{g \in G L(n+1, \mathbb{H}): A^{*} J A=J\right\} .
$$

Let $g \in \operatorname{Sp}(n, 1)$. Then $g$ and $g^{-1}$ are of the following forms:

$$
g=\left(\begin{array}{ccc}
a & \zeta^{*} & b \\
\alpha & A & \beta \\
c & \delta^{*} & d
\end{array}\right), \quad g^{-1}=\left(\begin{array}{ccc}
\bar{d} & \beta^{*} & \bar{b} \\
\delta & A^{*} & \zeta \\
\bar{c} & \alpha^{*} & \bar{a}
\end{array}\right),
$$


where $a, b, c, d \in \mathbb{H}, A$ is an $(n-1) \times(n-1)$ matrix over $\mathbb{H}$, and $\alpha, \beta, \zeta, \delta$ are column vectors in $\mathbb{H}^{n-1}$.

Following Section 2 of [8], let

$$
V_{0}=\left\{\mathbf{z} \in \mathbb{H}^{n, 1} \backslash\{0\}:\langle\mathbf{z}, \mathbf{z}\rangle=0\right\}, V_{-}=\left\{\mathbf{z} \in \mathbb{H}^{n, 1}:\langle\mathbf{z}, \mathbf{z}\rangle<0\right\} .
$$

Let $\mathbb{P}: \mathbb{H}^{n, 1} \backslash\{0\} \longrightarrow \mathbb{H}^{n}$ be the right projection onto $\mathbb{H}$-projective space. If $z_{n+1} \neq 0$ then $\mathbb{P}$ is given by

$$
\mathbb{P}\left(z_{1}, \ldots, z_{n}, z_{n+1}\right)^{T}=\left(z_{1} z_{n+1}^{-1}, \cdots, z_{n} z_{n+1}^{-1}\right)^{T} \in \mathbb{H}^{n}
$$

We also define

$$
\mathbb{P}\left(z_{1}, 0, \ldots, 0,0\right)^{T}=\infty, \mathbb{P}\left(0,0, \ldots, 0, z_{n+1}\right)^{T}=o .
$$

The Siegel domain model of the quaternionic hyperbolic $n$-space is defined to be $\mathbf{H}_{\mathbb{H}}^{n}=\mathbb{P}\left(V_{-}\right)$with the boundary $\partial \mathbf{H}_{\mathbb{H}}^{n}=\mathbb{P}\left(V_{0}\right)$. We mention that $g \in \operatorname{Sp}(n, 1)$ acts on $\mathbf{H}_{\mathbb{H}}^{n} \cup \partial \mathbf{H}_{\mathbb{H}}^{n}$ as $g(z)=\mathbb{P} g \mathbb{P}^{-1}(z)$. The Bergman metric on $\mathbf{H}_{\mathbb{H}}^{n}$ is given by the distance formula

$$
\cosh ^{2} \frac{\rho(z, w)}{2}=\frac{\langle\mathbf{z}, \mathbf{w}\rangle\langle\mathbf{w}, \mathbf{z}\rangle}{\langle\mathbf{z}, \mathbf{z}\rangle\langle\mathbf{w}, \mathbf{w}\rangle}, \quad \text { where } z, w \in \mathbf{H}_{\mathbb{H}}^{n}, \quad \mathbf{z} \in \mathbb{P}^{-1}(z), \mathbf{w} \in \mathbb{P}^{-1}(w)
$$

The holomorphic isometry group of $\mathbf{H}_{\mathbb{H}}^{n}$ is $\operatorname{PSp}(n, 1)=\operatorname{Sp}(n, 1) / \pm I_{n+1}$.

The standard lift of a finite point $z \in \overline{\mathbf{H}_{\mathbb{H}}^{n}}$ is

$$
\hat{\mathbf{z}}=\left(\begin{array}{c}
z \\
1
\end{array}\right) \in \mathbb{P}^{-1}(z)
$$

and the standard lift of $\infty$ is

$$
\hat{\infty}=(-1,0, \cdots, 0)^{T} .
$$

We will follow the above convention without any other statements in the sequel.

We close this section with the proof of Propositions 1.111.2.

Proof of Proposition 1.1. Since $\operatorname{Sp}(n, 1)$ acts transitively on $\mathbb{H}$-lines in $V_{-}$and doubly transitively on $\mathbb{H}$-lines in $V_{0}$, and each isometry of $\operatorname{Sp}(n, 1)$ preserves the Hermitian form, we only need to consider the following two cases.

Firstly, we assume that $\mathbf{z}=(0, \cdots, 0,1)^{T} \in V_{0}$ and $\mathbf{w}=\left(w_{1}, w_{2}, \cdots, w_{n+1}\right)^{T} \in V_{0} \cup V_{-}$. Then $\langle\mathbf{w}, \mathbf{w}\rangle=\overline{w_{1}} w_{n+1}+\overline{w_{n+1}} w_{1}+\sum_{i=2}^{n}\left|w_{i}\right|^{2} \leq 0$ and $\langle\mathbf{z}, \mathbf{w}\rangle=\overline{w_{1}}$. If $w_{1}=0$ then we see $w_{i}=0$ for $i=2, \cdots, n$ and so $\mathbf{w}=\mathbf{z} w_{n+1}$.

Secondly, we assume that $\mathbf{z}=(-1,0, \cdots, 0,1)^{T} \in V_{-}$. Then $\langle\mathbf{z}, \mathbf{w}\rangle=\overline{w_{1}}-\overline{w_{n+1}}$. Since $\langle\mathbf{w}, \mathbf{w}\rangle \leq 0$ we have $\overline{w_{1}} \neq \overline{w_{n+1}}$, which implies that $\langle\mathbf{z}, \mathbf{w}\rangle \neq 0$.

Proof of Proposition 1.2. By properties of quaternions, we can verify that

$$
\begin{gathered}
\left\langle\mathbf{p}_{1} \lambda_{1}, \mathbf{p}_{2} \lambda_{2}, \mathbf{p}_{3} \lambda_{3}\right\rangle=\left|\lambda_{2} \lambda_{3}\right|^{2} \overline{\lambda_{1}}\left\langle\mathbf{p}_{1}, \mathbf{p}_{2}, \mathbf{p}_{3}\right\rangle \lambda_{1}, \\
\Re\left(\left\langle\mathbf{p}_{1}, \mathbf{p}_{2}, \mathbf{p}_{3}\right\rangle\right)=\Re\left(\left\langle\mathbf{p}_{2}, \mathbf{p}_{1}\right\rangle\left\langle\mathbf{p}_{3}, \mathbf{p}_{2}\right\rangle\left\langle\mathbf{p}_{1}, \mathbf{p}_{3}\right\rangle\right)=\Re\left(\left\langle\mathbf{p}_{2}, \mathbf{p}_{3}, \mathbf{p}_{1}\right\rangle\right)=\Re\left(\left\langle\mathbf{p}_{3}, \mathbf{p}_{1}, \mathbf{p}_{2}\right\rangle\right)
\end{gathered}
$$

and

$$
\Re\left(\left\langle\mathbf{p}_{1}, \mathbf{p}_{2}, \mathbf{p}_{3}\right\rangle\right)=\Re\left(\overline{\left\langle\mathbf{p}_{1}, \mathbf{p}_{2}, \mathbf{p}_{3}\right\rangle}\right)=\Re\left(\left\langle\mathbf{p}_{2}, \mathbf{p}_{1}, \mathbf{p}_{3}\right\rangle\right) .
$$

These equalities imply that the sign of $\Re\left(\left\langle\mathbf{p}_{1}, \mathbf{p}_{2}, \mathbf{p}_{3}\right\rangle\right)$ is independent of the choice of the lifts $\mathbf{p}_{1}, \mathbf{p}_{2}, \mathbf{p}_{3}$ and invariant under permutations of the points $\mathbf{p}_{1}, \mathbf{p}_{2}, \mathbf{p}_{3}$. Due to fact that the sign of $\Re\left(\left\langle\mathbf{p}_{1}, \mathbf{p}_{2}, \mathbf{p}_{3}\right\rangle\right)$ is 
invariant under the action of isometries in $\operatorname{Sp}(n, 1)$, by the transitivity of $\operatorname{Sp}(n, 1)$ on $\overline{\mathbf{H}_{\mathbb{H}}^{n}}$, we need only to consider the following two cases.

Case (1): One of $p_{1}, p_{2}, p_{3}$ lies on $\partial \mathbf{H}_{\mathbb{H}}^{n}$.

We may assume that $p_{1}=\infty$ and $p_{2}=\left(u_{1}, \cdots, u_{n}\right)^{T}, p_{3}=\left(r_{1}, \cdots, r_{n}\right)^{T} \in \overline{\mathbf{H}_{\mathbb{H}}^{n}}$. Note that

$$
\left\langle\hat{\mathbf{p}_{1}}, \hat{\mathbf{p}_{2}}, \hat{\mathbf{p}_{3}}\right\rangle=r_{1}+\overline{u_{1}}+\sum_{i=2}^{n} \overline{u_{i}} r_{i}
$$

and $-\Re\left(u_{1}\right) \geq \frac{1}{2} \sum_{i=2}^{n}\left|u_{i}\right|^{2}$ and $-\Re\left(r_{1}\right) \geq \frac{1}{2} \sum_{i=2}^{n}\left|r_{i}\right|^{2}$. Therefore

$$
\begin{aligned}
\Re\left(\left\langle\hat{\mathbf{p}_{1}}, \hat{\mathbf{p}_{2}}, \hat{\mathbf{p}_{3}}\right\rangle\right) & =\Re\left(u_{1}\right)+\Re\left(r_{1}\right)+\Re\left(\sum_{i=2}^{n} \overline{u_{i}} r_{i}\right) \\
& \leq \Re\left(u_{1}\right)+\Re\left(r_{1}\right)+\sqrt{\sum_{i=2}^{n}\left|u_{i}\right|^{2} \sum_{i=2}^{n}\left|r_{i}\right|^{2}} \\
& \leq \Re\left(u_{1}\right)+\Re\left(r_{1}\right)+2 \sqrt{\Re\left(-u_{1}\right) \Re\left(-r_{1}\right)} \\
& =-\left(\sqrt{-\Re\left(u_{1}\right)}-\sqrt{-\Re\left(r_{1}\right)}\right)^{2} \leq 0 .
\end{aligned}
$$

Case (2): All three points $p_{1}, p_{2}, p_{3}$ lie in $\mathbf{H}_{\mathbb{H}}^{n}$.

We may assume that $p_{1}=(-1,0, \cdots, 0)^{T}$ and $p_{2}=\left(u_{1}, \cdots, u_{n}\right)^{T}, p_{3}=\left(r_{1}, \cdots, r_{n}\right)^{T}$. Then

$$
-\Re\left(u_{1}\right)>\frac{1}{2} \sum_{i=2}^{n}\left|u_{i}\right|^{2},-\Re\left(r_{1}\right)>\frac{1}{2} \sum_{i=2}^{n}\left|r_{i}\right|^{2}
$$

and

$$
\left\langle\hat{\mathbf{p}_{1}}, \hat{\mathbf{p}_{2}}, \hat{\mathbf{p}_{3}}\right\rangle=\left(u_{1}-1\right)\left(r_{1}+\overline{u_{1}}+\sum_{i=2}^{n} \overline{u_{i}} r_{i}\right)\left(\overline{r_{1}}-1\right) .
$$

For simplicity, let $\lambda=\sum_{i=2}^{n} \bar{u}_{i} r_{i}$. Then we have

$$
|\lambda| \leq 2 \sqrt{\Re\left(-u_{1}\right) \Re\left(-r_{1}\right)}
$$

and

$$
\begin{aligned}
& \Re\left(\left(r_{1}+\overline{u_{1}}\right)\left(\overline{r_{1}}-1\right)\left(u_{1}-1\right)\right)=-\left|r_{1}+\overline{u_{1}}\right|^{2}+\left(\left|r_{1}\right|^{2}+1\right) \Re\left(u_{1}\right)+\left(\left|u_{1}\right|^{2}+1\right) \Re\left(r_{1}\right) \\
= & -\left|r_{1}+\overline{u_{1}}\right|^{2}+4 \Re\left(u_{1}\right) \Re\left(r_{1}\right)+\left(\left|r_{1}\right|^{2}-2 \Re\left(r_{1}\right)+1\right) \Re\left(u_{1}\right)+\left(\left|u_{1}\right|^{2}-2 \Re\left(u_{1}\right)+1\right) \Re\left(r_{1}\right) \\
= & -\left|r_{1}-u_{1}\right|^{2}+\left|\overline{r_{1}}-1\right|^{2} \Re\left(u_{1}\right)+\left|u_{1}-1\right|^{2} \Re\left(r_{1}\right) .
\end{aligned}
$$

Therefore

$$
\begin{aligned}
\Re\left(\left\langle\hat{\mathbf{p}_{1}}, \hat{\mathbf{p}_{2}}, \hat{\mathbf{p}_{3}}\right\rangle\right) & =\Re\left(\left(r_{1}+\overline{u_{1}}+t\right)\left(\overline{r_{1}}-1\right)\left(u_{1}-1\right)\right) \\
& =\Re\left(\left(r_{1}+\overline{u_{1}}\right)\left(\overline{r_{1}}-1\right)\left(u_{1}-1\right)\right)+\Re\left(t\left(\overline{r_{1}}-1\right)\left(u_{1}-1\right)\right) \\
& \leq \Re\left(\left(r_{1}+\overline{u_{1}}\right)\left(\overline{r_{1}}-1\right)\left(u_{1}-1\right)\right)+|t|\left|\left(\overline{r_{1}}-1\right)\left(u_{1}-1\right)\right| \\
& \leq \Re\left(\left(r_{1}+\overline{u_{1}}\right)\left(\overline{r_{1}}-1\right)\left(u_{1}-1\right)\right)+2 \sqrt{\Re\left(-u_{1}\right) \Re\left(-r_{1}\right)}\left|\left(\overline{r_{1}}-1\right)\left(u_{1}-1\right)\right| \\
& =-\left|r_{1}-u_{1}\right|^{2}-\left(\left|\overline{r_{1}}-1\right| \overline{\Re\left(-u_{1}\right)}-\left|u_{1}-1\right| \sqrt{\Re\left(-r_{1}\right)}\right)^{2} \\
& \leq 0 .
\end{aligned}
$$




\section{Quaternionic geometric invariants}

\subsection{Quaternionic cros-ratios}

The complex cross-ratio given by (11) is independent of the choice of $\mathbf{p}_{i}$. This property enables one to use the cross-ratios either in $\partial \mathbf{H}_{\mathbb{C}}^{n}$ or $V_{0}$ freely. In an inner product space, the concept of cross-ratio always stems from its inner products. Due to the non-commutativity of quaternions, $\overline{\mathbf{H}_{\mathbb{H}}^{n}}$ and $V_{0} \cup V_{-}$are two different worlds for the concept of quaternionic cross-ratio. In this subsection, we define two quaternionic cross-ratios. The first one is defined on $V_{0} \cup V_{-}$and the second one is defined on $\overline{\mathbf{H}_{\mathbb{H}}^{n}}$.

Let $\mathbf{p}=\left(\mathbf{p}_{1}, \mathbf{p}_{2}, \mathbf{p}_{3}, \mathbf{p}_{4}\right)$ be a quadruple of points in $V_{0} \cup V_{-}$, we define

$$
\mathbb{P}(\mathbf{p})=\left(\mathbb{P}\left(\mathbf{p}_{1}\right), \mathbb{P}\left(\mathbf{p}_{2}\right), \mathbb{P}\left(\mathbf{p}_{3}\right), \mathbb{P}\left(\mathbf{p}_{4}\right)\right) \in\left(\overline{\mathbf{H}_{\mathbb{H}}^{n}}\right)^{4} .
$$

Definition 3.1. Let $\mathbf{p}=\left(\mathbf{p}_{1}, \mathbf{p}_{2}, \mathbf{p}_{3}, \mathbf{p}_{4}\right)$ be a quadruple of points in $V_{0} \cup V_{-}$such that $\mathbb{P}(\mathbf{p})$ are pairwise distinct points in $\overline{\mathbf{H}_{\mathbb{H}}^{n}}$. The quaternionic cross-ratio of $\mathbf{p}=\left(\mathbf{p}_{1}, \mathbf{p}_{2}, \mathbf{p}_{3}, \mathbf{p}_{4}\right)$ in $V_{0} \cup V_{-}$is defined as

$$
\mathbb{X}(\mathbf{p})=\mathbb{X}\left(\mathbf{p}_{1}, \mathbf{p}_{2}, \mathbf{p}_{3}, \mathbf{p}_{4}\right):=\left\langle\mathbf{p}_{3}, \mathbf{p}_{1}\right\rangle\left\langle\mathbf{p}_{3}, \mathbf{p}_{2}\right\rangle^{-1}\left\langle\mathbf{p}_{4}, \mathbf{p}_{2}\right\rangle\left\langle\mathbf{p}_{4}, \mathbf{p}_{1}\right\rangle^{-1}
$$

Proposition 1.1 implies that the definition above is well defined. We mention that the quaternionic cross-ratio above has been used by Platis to obtain the Ptolemaean inequality in the quaternionic hyperbolic space [20].

Observe that, for nonzero quaternions $\lambda_{i}, i=1, \cdots, 4$,

$$
\mathbb{X}\left(\mathbf{p}_{1} \lambda_{1}, \mathbf{p}_{2} \lambda_{2}, \mathbf{p}_{3} \lambda_{3}, \mathbf{p}_{4} \lambda_{4}\right)=\bar{\lambda}_{1} \mathbb{X}\left(\mathbf{p}_{1}, \mathbf{p}_{2}, \mathbf{p}_{3}, \mathbf{p}_{4}\right) \bar{\lambda}_{1}^{-1}
$$

The above equality is dominant among the properties of quaternionic cross-ratio in $V_{0} \cup V_{-}$.

Definition 3.2. Let $\mathfrak{p}=\left(p_{1}, p_{2}, p_{3}, p_{4}\right)$ be any quadruple of pairwise distinct points in $\overline{\mathbf{H}_{\mathbb{H}}^{n}}$. The quaternionic cross-ratio of $\mathfrak{p}=\left(p_{1}, p_{2}, p_{3}, p_{4}\right)$ in $\overline{\mathbf{H}_{\mathbb{H}}^{n}}$ is defined as

$$
\mathbb{X}(\mathfrak{p})=\mathbb{X}\left(p_{1}, p_{2}, p_{3}, p_{4}\right):=\mathbb{X}\left(\hat{\mathbf{p}}_{1}, \hat{\mathbf{p}}_{2}, \hat{\mathbf{p}}_{3}, \hat{\mathbf{p}}_{4}\right),
$$

where $\hat{\mathbf{p}}_{i}$ is the standard lift of $p_{i}$.

We mention that the restriction of Definition 3.2 on $\partial \mathbf{H}_{\mathbb{C}}^{n}$ is identical with the Korányi-Reimann complex cross-ratio [16]. We are now ready to obtain some properties of quaternionic cross-ratios.

Proposition 3.1. Let $\mathfrak{p}=\left(p_{1}, p_{2}, p_{3}, p_{4}\right)$ be any quadruple of pairwise distinct points in $\overline{\mathbf{H}_{\mathbb{H}}^{n}}$ with lifts $\mathbf{p}_{1}, \mathbf{p}_{2}, \mathbf{p}_{3}, \mathbf{p}_{4}$ and $h \in \operatorname{Sp}(n, 1)$. Then

$$
\Re(\mathbb{X}(\mathfrak{p}))=\Re(\mathbb{X}(\mathbf{p}))=\Re\left(\mathbb{X}\left(h\left(p_{1}\right), h\left(p_{2}\right), h\left(p_{3}\right), h\left(p_{4}\right)\right)\right) ;
$$

(ii) $\quad|\mathbb{X}(\mathfrak{p})|=|\mathbb{X}(\mathbf{p})|=\left|\mathbb{X}\left(h\left(p_{1}\right), h\left(p_{2}\right), h\left(p_{3}\right), h\left(p_{4}\right)\right)\right|$.

Proof. Noting that $\mathbf{p}_{i}=\hat{\mathbf{p}}_{i} \lambda_{i}$ for some $\lambda_{i} \neq 0$ and the standard lift of $h\left(p_{i}\right)$ can be expressed as $h \hat{\mathbf{p}}_{i} \mu_{i}, \mu_{i} \neq 0$, we have the following two equations:

$$
\begin{gathered}
\mathbb{X}(\mathbf{p})=\mathbb{X}\left(\hat{\mathbf{p}}_{1} \lambda_{1}, \hat{\mathbf{p}}_{2} \lambda_{2}, \hat{\mathbf{p}}_{3} \lambda_{3}, \hat{\mathbf{p}}_{4} \lambda_{4}\right)=\bar{\lambda}_{1} \mathbb{X}\left(\hat{\mathbf{p}}_{1}, \hat{\mathbf{p}}_{2}, \hat{\mathbf{p}}_{3}, \hat{\mathbf{p}}_{4}\right) \bar{\lambda}_{1}^{-1}=\bar{\lambda}_{1} \mathbb{X}(\mathfrak{p}) \bar{\lambda}_{1}^{-1}, \\
\mathbb{X}\left(h\left(p_{1}\right), h\left(p_{2}\right), h\left(p_{3}\right), h\left(p_{4}\right)\right)=\mathbb{X}\left(h \hat{\mathbf{p}}_{1} \mu_{1}, h \hat{\mathbf{p}}_{2} \mu_{2}, h \hat{\mathbf{p}}_{3} \mu_{3}, h \hat{\mathbf{p}}_{4} \mu_{4}\right)=\overline{\mu_{1}} \mathbb{X}(\mathfrak{p}) \bar{\mu}_{1}^{-1}
\end{gathered}
$$

The two equations above conclude the proof. 
We mention that those properties of quaternionic cross-ratio has been used in [6, 15] to obtain the generalized Jørgensen's inequalities in the quaternionic hyperbolic geometry.

The following proposition displays the relationships of quaternionic cross-ratios of a given quadruple under permutations of its points. One should compare them with analogous results in [13, Section 7.2] and [20]. The proof of the following proposition is by direct computations.

Proposition 3.2. $\quad$ (i) $\mathbb{X}\left(p_{1}, p_{2}, p_{3}, p_{4}\right)=\mathbb{X}\left(p_{1}, p_{2}, p_{4}, p_{3}\right)^{-1}$;

(ii) $\mathbb{X}\left(p_{1}, p_{2}, p_{3}, p_{4}\right) \sim \mathbb{X}\left(p_{2}, p_{1}, p_{3}, p_{4}\right)^{-1}$

(iii) $\mathbb{X}\left(p_{1}, p_{2}, p_{3}, p_{4}\right) \sim \mathbb{X}\left(p_{2}, p_{1}, p_{4}, p_{3}\right) \sim \mathbb{X}\left(p_{3}, p_{4}, p_{1}, p_{2}\right) \sim \mathbb{X}\left(p_{4}, p_{3}, p_{2}, p_{1}\right) ;$

(iv)

$$
\begin{aligned}
\left|\mathbb{X}\left(p_{1}, p_{2}, p_{3}, p_{4}\right) \mathbb{X}\left(p_{1}, p_{4}, p_{2}, p_{3}\right) \mathbb{X}\left(p_{1}, p_{3}, p_{4}, p_{2}\right)\right| & =\left|\mathbb{X}\left(p_{1}, p_{2}, p_{3}, p_{4}\right) \mathbb{X}\left(p_{4}, p_{2}, p_{1}, p_{3}\right) \mathbb{X}\left(p_{3}, p_{2}, p_{4}, p_{1}\right)\right| \\
& =\left|\mathbb{X}\left(p_{1}, p_{2}, p_{3}, p_{4}\right) \mathbb{X}\left(p_{4}, p_{2}, p_{3}, p_{1}\right) \mathbb{X}\left(p_{4}, p_{1}, p_{3}, p_{2}\right)\right| \\
& =\left|\mathbb{X}\left(p_{1}, p_{2}, p_{3}, p_{4}\right) \mathbb{X}\left(p_{2}, p_{3}, p_{1}, p_{4}\right) \mathbb{X}\left(p_{3}, p_{1}, p_{2}, p_{4}\right)\right| \\
& =1 .
\end{aligned}
$$

\subsection{Distance formulas}

We can relate the Bergman metric with the quaternionic cross-ratio as follows. We mention that analogous results in the 2- and the 4-dimensional unit ball have been obtained in [2, p133] and in [3, Section 5].

Proposition 3.3. Let $z, w \in \mathbf{H}_{\mathbb{H}}^{n}$ and $\gamma_{z w}$ be the geodesic connecting $z$ and $w$ with endpoints $u$ and $v$ in $\partial \mathbf{H}_{\mathbb{H}}^{n}$. Then

$$
\rho(z, w)=|\log (\mathbb{X}(z, u, w, v)-1)| .
$$

Proof. As in [6], for $u, v \in \partial \mathbf{H}_{\mathbb{H}}^{n}$ with lifts $\mathbf{u}$ and $\mathbf{v}$ such that $\langle\mathbf{u}, \mathbf{v}\rangle=-1$, the geodesic $\gamma_{u v}$ in $\mathbf{H}_{\mathbb{H}}^{n}$ with endpoints $u$ and $v$ parameterized by arc length $t$ is given by $\mathbb{P}(\gamma(t))$, where $\gamma(t)=e^{\frac{t}{2}} \mathbf{u}+e^{-\frac{t}{2}} \mathbf{v}$. Hence we can choose the lifts $\mathbf{z}, \mathbf{w}$ of $z, w$ as

$$
\mathbf{z}=e^{\frac{t}{2}} \mathbf{u}+e^{\frac{-t}{2}} \mathbf{v}, \mathbf{w}=e^{\frac{k}{2}} \mathbf{u}+e^{\frac{-k}{2}} \mathbf{v} .
$$

In this setting, $\rho(z, w)=|t-k|$ and $\mathbb{X}(z, u, w, v)=\mathbb{X}(\mathbf{z}, \mathbf{u}, \mathbf{w}, \mathbf{v})=1+e^{k-t}$.

As in [13, by Proposition 1.1, for $u, v \in \partial \mathbf{H}_{\mathbb{H}}^{n}$ and $z \in \mathbf{H}_{\mathbb{H}}^{n}$, the following quaternion

$$
\eta(u, v, z)=\langle\hat{\mathbf{u}}, \hat{\mathbf{z}}\rangle\langle\hat{\mathbf{u}}, \hat{\mathbf{v}}\rangle^{-1}\langle\hat{\mathbf{z}}, \hat{\mathbf{v}}\rangle\langle\hat{\mathbf{z}}, \hat{\mathbf{z}}\rangle^{-1}
$$

is well defined. By abuse of notation, one can view $\eta(u, v, z)$ in form as

$$
\eta(u, v, z)=\mathbb{X}(\hat{\mathbf{z}}, \hat{\mathbf{v}}, \hat{\mathbf{u}}, \hat{\mathbf{z}})=\mathbb{X}(z, v, u, z) .
$$

From this we know that the real part and the modulus of $\eta(u, v, z)$ are well defined in the quaternionic setting.

By repeating almost verbatim the arguments used for the complex case in Propositions 7.1 and 7.6 in [17], we obtain the following result. 
Proposition 3.4. Let $z \in \mathbf{H}_{\mathbb{H}}^{n}, u, v \in \partial \mathbf{H}_{\mathbb{H}}^{n}$ and $\gamma_{u v}$ be the geodesic connecting $u$ and $v$. Then the hyperbolic distance $\rho\left(\gamma_{u v}, z\right)$ from $z$ to $\gamma$ is given by

$$
\cosh ^{2}\left(\frac{\rho\left(\gamma_{u v}, z\right)}{2}\right)=|\eta(u, v, z)|+\Re(\eta(u, v, z))
$$

and the hyperbolic distance $\rho\left(L_{u v}, z\right)$ from $z$ to $L$ is given by

$$
\cosh ^{2}\left(\frac{\rho\left(L_{u v}, z\right)}{2}\right)=2 \Re(\eta(u, v, z)) .
$$

Let $z \in \mathbf{H}_{\mathbb{H}}^{n}, u, v \in \partial \mathbf{H}_{\mathbb{H}}^{n}$ with lifts $\mathbf{z}, \mathbf{u}$ and $\mathbf{v}$ such that $\langle\mathbf{u}, \mathbf{v}\rangle=-1$. By Proposition 3.4, the orthogonal projection $\gamma_{u v}(z)$ of $z \in \mathbf{H}_{\mathbb{H}}^{n}$ to the geodesic $\gamma_{u v}$ endowed with hyperbolic distance $\rho$ is given by

$$
\gamma_{u v}(z)=\mathbb{P}\left(e^{\frac{t}{2}} \mathbf{u}+e^{-\frac{t}{2}} \mathbf{v}\right),
$$

where $e^{t}=\left|\frac{\langle\mathbf{v}, \mathbf{z}\rangle}{\langle\mathbf{u}, \mathbf{z}\rangle}\right|$ and $\mathbf{z}$ is an arbitrary lift of $z$.

We need the following formula of hyperbolic distance form $z \in \mathbf{H}_{\mathbb{H}}^{n}$ to the the quaternionic line $L_{u w}$ spanned by $w \in \overline{\mathbf{H}_{\mathbb{H}}^{n}}$ and $u \in \partial \mathbf{H}_{\mathbb{H}}^{n}$ later.

Proposition 3.5. Let $z \in \mathbf{H}_{\mathbb{H}}^{n}, u \in \partial \mathbf{H}_{\mathbb{H}}^{n}$ and $v \in \mathbf{H}_{\mathbb{H}}^{n}$. Then

$$
\cosh ^{2}\left(\frac{\rho\left(L_{u v}, z\right)}{2}\right)=2 \Re(\eta(z, u, v))-\frac{|\langle\mathbf{u}, \mathbf{z}\rangle|^{2}\langle\mathbf{v}, \mathbf{v}\rangle}{|\langle\mathbf{u}, \mathbf{v}\rangle|^{2}\langle\mathbf{z}, \mathbf{z}\rangle}
$$

and

$$
\cosh ^{2}\left(\frac{\rho\left(\gamma_{u v}, z\right)}{2}\right)=\left|\mathbb{X}(\mathbf{z}, \mathbf{v}, \mathbf{u}, \mathbf{z})-\frac{1}{2} \frac{|\langle\mathbf{u}, \mathbf{z}\rangle|^{2}\langle\mathbf{v}, \mathbf{v}\rangle}{|\langle\mathbf{u}, \mathbf{v}\rangle|^{2}\langle\mathbf{z}, \mathbf{z}\rangle}\right|+\Re(\eta(z, u, v))-\frac{1}{2} \frac{|\langle\mathbf{u}, \mathbf{z}\rangle|^{2}\langle\mathbf{v}, \mathbf{v}\rangle}{|\langle\mathbf{u}, \mathbf{v}\rangle|^{2}\langle\mathbf{z}, \mathbf{z}\rangle}
$$

where $\mathbf{z}, \mathbf{u}$ and $\mathbf{v}$ and are lifts of $z, u, v$, respectively.

Proof. Let $w$ be the other endpoint of the geodesic $\gamma_{u v}$ and $\mathbf{u}$ and $\mathbf{w}$ be lifts of $u$ and $w$ such that $\langle\mathbf{u}, \mathbf{w}\rangle=-1$. Then there exist a $t \in \mathbb{R}$ and a lift $\mathbf{v}$ of $v$ such that $\mathbf{v}=e^{\frac{t}{2}} \mathbf{u}+e^{-\frac{t}{2}} \mathbf{w}$. Therefore

$$
\mathbf{w}=e^{\frac{t}{2}} \mathbf{v}-e^{t} \mathbf{u},\langle\mathbf{u}, \mathbf{v}\rangle=-e^{-\frac{t}{2}},\langle\mathbf{v}, \mathbf{v}\rangle=-2
$$

Let $\mu=\mathbb{X}(\mathbf{z}, \mathbf{w}, \mathbf{u}, \mathbf{z})$. Then by (27) we can express $\mu$ as

$$
\begin{aligned}
\mu & =\langle\mathbf{u}, \mathbf{z}\rangle\langle\mathbf{u}, \mathbf{v}\rangle^{-1}\langle\mathbf{z}, \mathbf{v}\rangle\langle\mathbf{z}, \mathbf{z}\rangle^{-1}-\frac{1}{2} \frac{|\langle\mathbf{u}, \mathbf{z}\rangle|^{2}\langle\mathbf{v}, \mathbf{v}\rangle}{|\langle\mathbf{u}, \mathbf{v}\rangle|^{2}\langle\mathbf{z}, \mathbf{z}\rangle} \\
& =\mathbb{X}(\mathbf{z}, \mathbf{v}, \mathbf{u}, \mathbf{z})-\frac{1}{2} \frac{|\langle\mathbf{u}, \mathbf{z}\rangle|^{2}\langle\mathbf{v}, \mathbf{v}\rangle}{|\langle\mathbf{u}, \mathbf{v}\rangle|^{2}\langle\mathbf{z}, \mathbf{z}\rangle}
\end{aligned}
$$

It is obvious that

$$
\cosh ^{2}\left(\frac{\rho\left(L_{u v}, z\right)}{2}\right)=\cosh ^{2}\left(\frac{\rho\left(L_{u w}, z\right)}{2}\right), \cosh ^{2}\left(\frac{\rho\left(\gamma_{u v}, z\right)}{2}\right)=\cosh ^{2}\left(\frac{\rho\left(\gamma_{u w}, z\right)}{2}\right) .
$$

Note that $|\mu|$ and $\Re(\mu)$ are independent of the choice of lifts $\mathbf{z}, \mathbf{u}, \mathbf{v}$. The result follows from Proposition 3.4 . 


\subsection{Quaternionic Cartan's angular invariant}

Recall from Section 1 that given a triple $\mathfrak{p}=\left(p_{1}, p_{2}, p_{3}\right)$ of pairwise distinct points in $\overline{\mathbf{H}_{\mathbb{H}}^{n}}$ with lifts $\mathbf{p}_{1}, \mathbf{p}_{2}, \mathbf{p}_{3}$, then

$$
\mathbb{A}_{\mathbb{H}}(\mathfrak{p})=\mathbb{A}_{\mathbb{H}}\left(p_{1}, p_{2}, p_{3}\right)=\arccos \frac{\Re\left(-\left\langle\mathbf{p}_{1}, \mathbf{p}_{2}, \mathbf{p}_{3}\right\rangle\right)}{\left|\left\langle\mathbf{p}_{1}, \mathbf{p}_{2}, \mathbf{p}_{3}\right\rangle\right|}
$$

is the quaternionic Cartan's angular invariant associated to $\mathfrak{p}$. This is the angle between the real axis and the radius vector of $\left\langle\mathbf{p}_{1}, \mathbf{p}_{2}, \mathbf{p}_{3}\right\rangle$ used by Apanasov and Kim [1].

As in the proof of Proposition 1.2, we have

$$
\mathbb{A}_{\mathbb{H}}(\mathfrak{p})=\arccos \frac{\Re\left(-\left\langle\mathbf{p}_{1} \lambda_{1}, \mathbf{p}_{2} \lambda_{2}, \mathbf{p}_{3} \lambda_{3}\right\rangle\right)}{\left|\left\langle\mathbf{p}_{1} \lambda_{1}, \mathbf{p}_{2} \lambda_{2}, \mathbf{p}_{3} \lambda_{3}\right\rangle\right|}
$$

and

$$
\mathbb{A}_{\mathbb{H}}\left(p_{1}, p_{2}, p_{3}\right)=\mathbb{A}_{\mathbb{H}}\left(p_{\iota(1)}, p_{\iota(2)}, p_{\iota(3)}\right),
$$

where $\iota$ is a permutation of $1,2,3$ and $\lambda_{i} \in \mathbb{H} \backslash\{0\}$. Therefore it makes sense to define

$$
\mathbb{A}_{\mathbb{H}}(\mathbf{p})=\mathbb{A}_{\mathbb{H}}\left(\mathbf{p}_{1}, \mathbf{p}_{2}, \mathbf{p}_{3}\right)=\arccos \frac{\Re\left(-\left\langle\mathbf{p}_{1}, \mathbf{p}_{2}, \mathbf{p}_{3}\right\rangle\right)}{\left|\left\langle\mathbf{p}_{1}, \mathbf{p}_{2}, \mathbf{p}_{3}\right\rangle\right|}
$$

We can verify that

$$
\mathbb{A}_{\mathbb{H}}(\mathbf{p})=\mathbb{A}_{\mathbb{H}}\left(\mathbf{p}_{1}, \mathbf{p}_{2}, \mathbf{p}_{3}\right)=\mathbb{A}_{\mathbb{H}}\left(\mathbf{p}_{1} \lambda_{1}, \mathbf{p}_{2} \lambda_{2}, \mathbf{p}_{3} \lambda_{3}\right)=\mathbb{A}_{\mathbb{H}}\left(\mathbf{p}_{\iota(1)} \lambda_{\iota(1)}, \mathbf{p}_{\iota(2)} \lambda_{\iota(2)}, \mathbf{p}_{\iota(3)} \lambda_{\iota(3)}\right)
$$

and

$$
\mathbb{A}_{\mathbb{H}}(\mathfrak{p})=\mathbb{A}_{\mathbb{H}}\left(g\left(p_{1}\right), g\left(p_{2}\right), g\left(p_{3}\right)\right), \forall g \in \operatorname{Sp}(n, 1) .
$$

The following properties are in [1] .

Proposition 3.6. Let $\mathfrak{p}=\left(p_{1}, p_{2}, p_{3}\right)$ be a triple of pairwise distinct points in $\partial \mathbf{H}_{\mathbb{H}}^{n}$. Then

(i) Three points $p_{1}, p_{2}, p_{3}$ lie in the same $\mathbb{R}$-circle if and only if $\mathbb{A}_{\mathbb{H}}(\mathfrak{p})=0$.

(ii) Three points $p_{1}, p_{2}, p_{3}$ lie in the boundary of an $\mathbb{H}$-line if and only if $\mathbb{A}_{\mathbb{H}}(\mathfrak{p})=\pi / 2$.

The quaternionic Cartan's angular invariant enjoys the following geometric interpretation. This geometric interpretation is a slight generalisation of Theorem 3.4 in [1].

Proposition 3.7. For distinct points $z, w \in \partial \mathbf{H}_{\mathbb{H}}^{n}$ and $r \in \overline{\mathbf{H}_{\mathbb{H}}^{n}}$, let $\Pi: \mathbf{H}_{\mathbb{H}}^{n} \rightarrow L_{z w}$ and $\Theta: \mathbf{H}_{\mathbb{H}}^{n} \rightarrow \gamma_{z w}$ be the orthogonal projections of the space $\mathbf{H}_{\mathbb{H}}^{n}$ endowed with hyperbolic distance $\rho$. Then

$$
\Theta \Pi r=\Theta r, \quad \tan \mathbb{A}_{\mathbb{H}}(z, w, r)=\sinh \left(\rho\left(\gamma_{z w}, \Pi r\right)\right)
$$

(ii) If $r \in \mathbf{H}_{\mathbb{H}}^{n}$, then we have

$$
\cosh \left(\frac{\rho\left(\gamma_{z w}, r\right)}{2}\right)=\cosh \left(\frac{\rho\left(L_{z w}, r\right)}{2}\right) \cosh \left(\frac{\rho\left(\gamma_{z w}, \Pi r\right)}{2}\right)
$$

and

$$
\tan \mathbb{A}_{\mathbb{H}}(z, w, r)=\frac{2 \sqrt{2} \cosh \left(\frac{\rho\left(\gamma_{z w}, r\right)}{2}\right) \sqrt{\cosh \rho\left(\gamma_{z w}, r\right)-\cosh \rho\left(L_{z w}, r\right)}}{1+\cosh \rho\left(L_{z w}, r\right)} .
$$


Proof. Since $\operatorname{Sp}(n, 1)$ acts doubly transitively on $\partial \mathbf{H}_{\mathbb{H}}^{n}$, we may assume that $z=o, w=\infty, r=$ $\left(r_{1}, \cdots, r_{n}\right)^{T} \in \overline{\mathbf{H}_{\mathbb{H}}^{n}}$. Then

$$
L_{z w}=\mathbb{P}(\{\hat{\mathbf{o}} \lambda+\hat{\boldsymbol{\infty}} \mu, \lambda, \mu, \in \mathbb{H}\}) \cap \overline{\mathbf{H}_{\mathbb{H}}^{n}}=\left\{(x, 0, \cdots, 0)^{T}: \Re(x) \leq 0\right\} .
$$

Therefore $\Pi r=\left(r_{1}, 0, \cdots, 0\right)^{T}$. It follows from (26) that

$$
\Theta \Pi r=\Theta r=\left(-\left|r_{1}\right|, 0, \cdots, 0\right) .
$$

Since $\langle\hat{\mathbf{o}}, \hat{\infty}, \hat{\mathbf{r}}\rangle=\overline{r_{1}}$, we have $\cos \mathbb{A}_{\mathbb{H}}(\mathfrak{p})=\frac{-\Re\left(r_{1}\right)}{\left|r_{1}\right|}$ and therefore

$$
\tan \mathbb{A}_{\mathbb{H}}(z, w, r)=\frac{\left|\Im\left(r_{1}\right)\right|}{-\Re\left(r_{1}\right)} .
$$

Noting that $\eta(o, \infty, \Pi r)=\frac{\overline{r_{1}}}{2 \Re\left(r_{1}\right)}$, by Proposition 3.4 we get

$$
\cosh ^{2}\left(\frac{\rho\left(\gamma_{z w}, \Pi r\right)}{2}\right)=\frac{\Re\left(r_{1}\right)-\left|r_{1}\right|}{2 \Re\left(r_{1}\right)} .
$$

Therefore

$$
\sinh ^{2}\left(\rho\left(\gamma_{z w}, \Pi r\right)\right)=\left[2 \cosh ^{2}\left(\frac{\rho\left(\gamma_{z w}, \Pi r\right)}{2}\right)-1\right]^{2}-1=\frac{\left|\Im\left(r_{1}\right)\right|^{2}}{\Re\left(r_{1}\right)^{2}} .
$$

This concludes the proof of (i).

If $r \in \mathbf{H}_{\mathbb{H}}^{n}$ then $2 \Re\left(r_{1}\right)+\sum_{i=2}^{n}\left|r_{i}\right|^{2}<0$ and

$$
\eta(o, \infty, r)=\frac{\overline{r_{1}}}{2 \Re\left(r_{1}\right)+\sum_{i=2}^{n}\left|r_{i}\right|^{2}} .
$$

It follows from Proposition 3.4 that

$$
\cosh ^{2}\left(\frac{\rho\left(\gamma_{z w}, r\right)}{2}\right)=\frac{\Re\left(r_{1}\right)-\left|r_{1}\right|}{2 \Re\left(r_{1}\right)+\sum_{i=2}^{n}\left|r_{i}\right|^{2}}, \quad \cosh ^{2}\left(\frac{\rho\left(L_{z w}, r\right)}{2}\right)=\frac{2 \Re\left(r_{1}\right)}{2 \Re\left(r_{1}\right)+\sum_{i=2}^{n}\left|r_{i}\right|^{2}} .
$$

Hence

$$
\cosh \left(\frac{\rho\left(\gamma_{z w}, r\right)}{2}\right)=\cosh \left(\frac{\rho\left(L_{z w}, r\right)}{2}\right) \cosh \left(\frac{\rho\left(\gamma_{z w}, \Pi r\right)}{2}\right)
$$

By (36) we get

$$
\sinh ^{2}\left(\rho\left(\gamma_{z w}, \Pi r\right)\right)=\frac{8 \cosh ^{2}\left(\frac{\rho\left(\gamma_{z w}, r\right)}{2}\right)\left(\cosh \rho\left(\gamma_{z w}, r\right)-\cosh \rho\left(L_{z w}, r\right)\right)}{\left(1+\cosh \rho\left(L_{z w}, r\right)\right)^{2}} .
$$

The above equation, together with (32) and (34), concludes the proof of (ii).

The following proposition relates the cyclic product of quaternionic cross-ratios to the quaternionic Cartan's angular invariant. One can compare it with analogous result of complex case in [13, p225].

\section{Proposition 3.8.}

$$
\mathbb{X}\left(p_{1}, p_{2}, p_{3}, p_{4}\right) \mathbb{X}\left(p_{1}, p_{4}, p_{2}, p_{3}\right) \mathbb{X}\left(p_{1}, p_{3}, p_{4}, p_{2}\right)=\exp \left(2 \mathbb{A}_{\mathbb{H}}\left(p_{2}, p_{3}, p_{4}\right) \mathbf{J}\right),
$$

where $\mathbf{J}$ is the imaginary part of $\mathbb{X}\left(p_{1}, p_{2}, p_{3}, p_{4}\right) \mathbb{X}\left(p_{1}, p_{4}, p_{2}, p_{3}\right) \mathbb{X}\left(p_{1}, p_{3}, p_{4}, p_{2}\right)$. 
Proof. Let $Y=\mathbb{X}\left(p_{1}, p_{2}, p_{3}, p_{4}\right) \mathbb{X}\left(p_{1}, p_{4}, p_{2}, p_{3}\right) \mathbb{X}\left(p_{1}, p_{3}, p_{4}, p_{2}\right)$. Then $|Y|=1$ and

$$
\begin{aligned}
Y= & \left\langle\hat{\mathbf{p}}_{3}, \hat{\mathbf{p}}_{1}\right\rangle\left\langle\hat{\mathbf{p}}_{3}, \hat{\mathbf{p}}_{2}\right\rangle^{-1}\left\langle\hat{\mathbf{p}}_{4}, \hat{\mathbf{p}}_{2}\right\rangle\left\langle\hat{\mathbf{p}}_{4}, \hat{\mathbf{p}}_{1}\right\rangle^{-1}\left\langle\hat{\mathbf{p}}_{2}, \hat{\mathbf{p}}_{1}\right\rangle\left\langle\hat{\mathbf{p}}_{2}, \hat{\mathbf{p}}_{4}\right\rangle^{-1}\left\langle\hat{\mathbf{p}}_{3}, \hat{\mathbf{p}}_{4}\right\rangle\left\langle\hat{\mathbf{p}}_{3}, \hat{\mathbf{p}}_{1}\right\rangle^{-1} \\
& \left\langle\hat{\mathbf{p}}_{4}, \hat{\mathbf{p}}_{1}\right\rangle\left\langle\hat{\mathbf{p}}_{4}, \hat{\mathbf{p}}_{3}\right\rangle^{-1}\left\langle\hat{\mathbf{p}}_{2}, \hat{\mathbf{p}}_{3}\right\rangle\left\langle\hat{\mathbf{p}}_{2}, \hat{\mathbf{p}}_{1}\right\rangle^{-1} .
\end{aligned}
$$

Hence

$$
\Re(Y)=\Re\left(\frac{\left\langle\hat{\mathbf{p}}_{2}, \hat{\mathbf{p}}_{3}, \hat{\mathbf{p}}_{4}\right\rangle^{2}}{\left|\left\langle\hat{\mathbf{p}}_{2}, \hat{\mathbf{p}}_{3}, \hat{\mathbf{p}}_{4}\right\rangle\right|^{2}}\right)
$$

Noting that

$$
\frac{-\left\langle\hat{\mathbf{p}}_{2}, \hat{\mathbf{p}}_{3}, \hat{\mathbf{p}}_{4}\right\rangle}{\left|\left\langle\hat{\mathbf{p}}_{2}, \hat{\mathbf{p}}_{3}, \hat{\mathbf{p}}_{4}\right\rangle\right|}=\exp \left(\mathbb{A}_{\mathbb{H}}\left(p_{2}, p_{3}, p_{4}\right) \mathbf{I}\right), \quad \mathbf{I}=\frac{-\Im\left(\left\langle\hat{\mathbf{p}}_{2}, \hat{\mathbf{p}}_{3}, \hat{\mathbf{p}}_{4}\right\rangle\right)}{\left|\Im\left(\left\langle\hat{\mathbf{p}}_{2}, \hat{\mathbf{p}}_{3}, \hat{\mathbf{p}}_{4}\right\rangle\right)\right|},
$$

we have

$$
\frac{\left\langle\hat{\mathbf{p}}_{2}, \hat{\mathbf{p}}_{3}, \hat{\mathbf{p}}_{4}\right\rangle^{2}}{\left|\left\langle\hat{\mathbf{p}}_{2}, \hat{\mathbf{p}}_{3}, \hat{\mathbf{p}}_{4}\right\rangle\right|^{2}}=\exp \left(2 \mathbb{A}_{\mathbb{H}}\left(p_{2}, p_{3}, p_{4}\right) \mathbf{I}\right)
$$

\section{The congruence class of triple of pairwise distinct points of $\overline{\mathbf{H}_{\mathbb{H}}^{n}}$}

Denote by

$$
G_{o, \infty}=\{g \in \operatorname{Sp}(n, 1): g(o)=o, g(\infty)=\infty\} .
$$

It follows from (18) that each $h \in G_{o, \infty}$ is of the form

$$
h=\operatorname{diag}\left(\mu, A, \bar{\mu}^{-1}\right),
$$

where $A \in \operatorname{Sp}(n-1)$. Moveover, if $|\mu|=1$ then $h$ is a boundary elliptic element fixing the geodesic $\gamma_{o \infty}$ pointwise.

We need the following lemma to prove Theorem 1.1 .

Lemma 4.1. Let $z=\left(z_{1}, \cdots, z_{n}\right)^{T}$ and $w=\left(w_{1}, \cdots, w_{n}\right)^{T}$ be points of $\overline{\mathbf{H}_{\mathbb{H}}^{n}}$. Then

(i) there exists an $h \in G_{o, \infty}$ such that $w=h(z)$ if and only if this exists a $\kappa>0$ such that

$$
\Re\left(w_{1}\right)=\kappa^{2} \Re\left(z_{1}\right), \quad\left|w_{1}\right|=\kappa^{2}\left|z_{1}\right|, \quad \sum_{i=2}^{n}\left|w_{i}\right|^{2}=\kappa^{2} \sum_{i=2}^{n}\left|z_{i}\right|^{2} ;
$$

(ii) there exists an elliptic element $h$ fixing the geodesic $\gamma_{o \infty}$ pointwise such that $w=h(z)$ if and only if

$$
\Re\left(w_{1}\right)=\Re\left(z_{1}\right), \quad\left|w_{1}\right|=\left|z_{1}\right|, \quad \sum_{i=2}^{n}\left|w_{i}\right|^{2}=\sum_{i=2}^{n}\left|z_{i}\right|^{2} .
$$

Proof. We first consider Case (i). Let $h=\operatorname{diag}\left(\mu, A, \bar{\mu}^{-1}\right)$, where $A \in \operatorname{Sp}(n-1)$. It follows from $w=h(z)$ that $h \hat{\mathbf{z}}=\hat{\mathbf{w}} \bar{\mu}^{-1}$, that is,

$$
\mu z_{1}=w_{1} \bar{\mu}^{-1}, A\left(z_{2}, \cdots, z_{n}\right)^{T}=\left(w_{2}, \cdots, w_{n}\right)^{T} \bar{\mu}^{-1} .
$$

Therefore the condition (37) holds. 
For sufficiency, if the condition (37) holds then there exist a unit quaternion $\lambda$ and an $A \in \operatorname{Sp}(n-1)$ such that

$$
\lambda\left(\kappa^{2} z_{1}\right) \lambda^{-1}=w_{1}, A\left(z_{2}, \cdots, z_{n}\right)^{T}=\left(w_{2}, \cdots, w_{n}\right)^{T} \frac{\lambda}{\kappa} .
$$

Therefore $h=\operatorname{diag}\left(\kappa \lambda, A, \frac{\lambda}{\kappa}\right) \in G_{o, \infty}$ is the desired isometry mapping $z$ to $w$.

Similarly we can prove Case (ii).

We now consider the congruence class of triple of pairwise distinct points of $\overline{\mathbf{H}_{\mathbb{H}}^{n}}$.

Proof of Theorem 1.1. The necessity is obvious. We mention that the geometric invariants in Theorem 1.1 are invariant under the conjugation by elements of $\operatorname{Sp}(n, 1)$. For sufficiency, we can choose $f, g \in$ $\operatorname{Sp}(n, 1)$ to map the geodesics connecting $p_{1}$ and $p_{2}, q_{1}$ and $q_{2}$ to $\gamma_{o \infty}$, respectively. By our condition, we can further assume that $f\left(p_{1}\right)=g\left(q_{1}\right)$ and $f\left(p_{2}\right)=g\left(q_{2}\right)$. Then we need to find an $h \in \operatorname{Sp}(n, 1)$ which fixes $f\left(p_{1}\right)$ and $f\left(p_{2}\right)$ and maps $f\left(p_{3}\right)$ to $g\left(q_{3}\right)$.

Let $p_{3}=\left(r_{1}, \cdots, r_{n}\right)^{T}, q_{3}=\left(z_{1}, \cdots, z_{n}\right)^{T} \in \overline{\mathbf{H}_{\mathbb{H}}^{n}}$. By the above normalisation, we only need to consider the following four specific cases.

Case (i) and Case (ii): $\mathfrak{p}=\left(o, \infty, p_{3}\right)$ and $\mathfrak{q}=\left(o, \infty, q_{3}\right), p_{3}, q_{3} \in \overline{\mathbf{H}_{\mathbb{H}}^{n}}$.

Since $\left\langle\hat{\mathbf{o}}, \hat{\infty}, \hat{\mathbf{p}}_{3}\right\rangle=\bar{r}_{1}$ and $\mathbb{A}_{\mathbb{H}}(\mathfrak{p})=\mathbb{A}_{\mathbb{H}}(\mathfrak{q})$, we get

$$
\frac{\Re\left(r_{1}\right)}{\left|r_{1}\right|}=\frac{\Re\left(z_{1}\right)}{\left|z_{1}\right|} .
$$

For Case (i), that is $p_{3}, q_{3} \in \partial \mathbf{H}_{\mathbb{H}}^{n}$, we have $-2 \Re\left(r_{1}\right)=\sum_{i=2}^{n}\left|r_{i}\right|^{2}$ and $-2 \Re\left(z_{1}\right)=\sum_{i=2}^{n}\left|z_{i}\right|^{2}$. Therefore the condition (37) holds.

For Case (ii), that is $p_{3}, q_{3} \in \mathbf{H}_{\mathbb{H}}^{n}$, we have $-2 \Re\left(r_{1}\right)>\sum_{i=2}^{n}\left|r_{i}\right|^{2}$ and $-2 \Re\left(z_{1}\right)>\sum_{i=2}^{n}\left|z_{i}\right|^{2}$. Since

$$
\eta\left(o, \infty, p_{3}\right)=\frac{\overline{r_{1}}}{2 \Re\left(r_{1}\right)+\sum_{i=2}^{n}\left|r_{i}\right|^{2}}
$$

and $\rho\left(L_{o \infty}, p_{3}\right)=\rho\left(L_{o \infty}, q_{3}\right)$, by Proposition 3.4 we have

$$
\frac{2 \Re\left(r_{1}\right)}{2 \Re\left(r_{1}\right)+\sum_{i=2}^{n}\left|r_{i}\right|^{2}}=\frac{2 \Re\left(z_{1}\right)}{2 \Re\left(z_{1}\right)+\sum_{i=2}^{n}\left|z_{i}\right|^{2}} .
$$

Thus the condition (37) holds. Lemma 4.1 concludes the proof of Cases (i) and (ii).

Remark 4.1. In Case (ii), by Proposition 3.7 we can use $\rho\left(\gamma_{p_{1} p_{2}}, p_{3}\right)=\rho\left(\gamma_{q_{1} q_{2}}, q_{3}\right)$ to replace the condition $\rho\left(L_{p_{1} p_{2}}, p_{3}\right)=\rho\left(L_{q_{1} q_{2}}, q_{3}\right)$. Let $\mathfrak{p}=\left(o, \infty, p_{3}\right)$ and $\mathfrak{q}=\left(o, \infty, q_{3}\right)$, where $p_{3}=\left(-1, \frac{\sqrt{6}}{2}\right) \in \mathbf{H}_{\mathbb{C}}^{2}$ and $q_{3}=(-1, \sqrt{2} \mathbf{i}) \in \partial \mathbf{H}_{\mathbb{C}}^{2}$. Though $\mathbb{A}_{\mathbb{H}}(\mathfrak{p})=\mathbb{A}_{\mathbb{H}}(\mathfrak{q})=0$, we can not map $p_{3}$ to $q_{3}$ by any $f \in \operatorname{Sp}(n, 1)$. This implies the condition $\rho\left(L_{p_{1} p_{2}}, p_{3}\right)=\rho\left(L_{q_{1} q_{2}}, q_{3}\right)$ is necessary.

Case (iii): $p_{1} \in \partial \mathbf{H}_{\mathbb{H}}^{n}, p_{2}, p_{3} \in \mathbf{H}_{\mathbb{H}}^{n}$.

Since $\operatorname{Sp}(n, 1)$ acts transitively on $\partial \mathbf{H}_{\mathbb{H}}^{n} \times \mathbf{H}_{\mathbb{H}}^{n}$, we may assume that $p_{1}=q_{1}=o$ and $p_{2}=q_{2}=$ $(-1,0, \cdots, 0)^{T}=\mathbb{P}(\hat{\infty}+\hat{\mathbf{o}})$. In this case the other end point of the geodesic connecting $o$ and $p_{2}$ is $\infty$. It follows from $\rho\left(L_{o \infty}, p_{3}\right)=\rho\left(L_{o \infty}, q_{3}\right)$ that

$$
\frac{\Re\left(r_{1}\right)}{2 \Re\left(r_{1}\right)+\sum_{i=2}^{n}\left|r_{i}\right|^{2}}=\frac{\Re\left(z_{1}\right)}{2 \Re\left(z_{1}\right)+\sum_{i=2}^{n}\left|z_{i}\right|^{2}} .
$$


By $\rho\left(p_{2}, p_{3}\right)=\rho\left(q_{2}, q_{3}\right)$ we get

$$
\frac{\left|r_{1}-1\right|^{2}}{2 \Re\left(r_{1}\right)+\sum_{i=2}^{n}\left|r_{i}\right|^{2}}=\frac{\left|z_{1}-1\right|^{2}}{2 \Re\left(z_{1}\right)+\sum_{i=2}^{n}\left|z_{i}\right|^{2}} .
$$

It follows from (41) and (42) that

$$
\frac{\sum_{i=2}^{n}\left|r_{i}\right|^{2}}{\Re\left(r_{1}\right)}=\frac{\sum_{i=2}^{n}\left|z_{i}\right|^{2}}{\Re\left(z_{1}\right)}, \frac{\Re\left(r_{1}\right)}{\left|1-r_{1}\right|^{2}}=\frac{\Re\left(z_{1}\right)}{\left|1-z_{1}\right|^{2}}, \frac{1+\left|r_{1}\right|^{2}}{\Re\left(r_{1}\right)}=\frac{1+\left|z_{1}\right|^{2}}{\Re\left(z_{1}\right)} .
$$

By Proposition 3.5] we have

$$
\cosh ^{2}\left(\frac{\rho\left(L_{o r}, p_{2}\right)}{2}\right)=1+\frac{\sum_{i=2}^{n}\left|r_{i}\right|^{2}}{2\left|r_{1}\right|^{2}}, \cosh ^{2}\left(\frac{\rho\left(L_{o q_{3}}, q_{2}\right)}{2}\right)=1+\frac{\sum_{i=2}^{n}\left|z_{i}\right|^{2}}{2\left|z_{1}\right|^{2}}
$$

Hence

$$
\frac{\sum_{i=2}^{n}\left|r_{i}\right|^{2}}{\left|r_{1}\right|^{2}}=\frac{\sum_{i=2}^{n}\left|z_{i}\right|^{2}}{\left|z_{1}\right|^{2}}
$$

By (43) and (44) we get $\Re\left(r_{1}\right)=\Re\left(z_{1}\right),\left|z_{1}\right|=\left|r_{1}\right|, \quad \sum_{i=2}^{n}\left|z_{i}\right|^{2}=\sum_{i=2}^{n}\left|r_{i}\right|^{2}$. Lemma 4.1 concludes the proof of Case (iii).

Remark 4.2. Since $\left\langle\hat{\mathbf{o}}, \hat{\mathbf{o}}+\hat{\infty}, \hat{\mathbf{p}}_{3}\right\rangle=\overline{r_{1}}-\left|r_{1}\right|^{2}$, the condition $\mathbb{A}_{\mathbb{H}}(\mathfrak{p})=\mathbb{A}_{\mathbb{H}}(\mathfrak{q})$ implies that

$$
\frac{\Re\left(r_{1}\right)-\left|r_{1}\right|^{2}}{\left|r_{1}\right|\left|1-r_{1}\right|}=\frac{\Re\left(z_{1}\right)-\left|z_{1}\right|^{2}}{\left|z_{1}\right|\left|1-z_{1}\right|} .
$$

Let $p_{3}=(-1+\mathbf{i}, \sqrt{6} / 2), q_{3}=(-2 / 5+\mathbf{i} / 5, \sqrt{15} / 5)$. Then the conditions (41), (42) and (45) hold for $\mathfrak{p}=\left(o, \mathbb{P}(\hat{\infty}+\hat{\mathbf{o}}), p_{3}\right)$ and $\mathfrak{q}=\left(o, \mathbb{P}(\hat{\infty}+\hat{\mathbf{o}}), q_{3}\right)$. If there exist an $h \in \operatorname{Sp}(2,1)$ mapping $\mathfrak{p}$ to $\mathfrak{q}$ then $h$ fixes $o$ and $\mathbb{P}(\hat{\infty}+\hat{\mathbf{o}})$. This implies that $h$ is a boundary elliptic element fixing the geodesic $\gamma_{o \infty}$ pointwise. However Lemma 4.1(ii) indicates that there exist not such an $h \in \operatorname{Sp}(2,1)$ for $-1 \neq-2 / 5$. This example shows that the quaternionic Cartan's angular invariant is not a good candidate for congruence class in this case.

Case (iv): $p_{1}, p_{2}, p_{3} \in \mathbf{H}_{\mathbb{H}}^{n}$

Mapping the geodesic $\gamma_{p_{1} p_{2}}$ and $\gamma_{q_{1} q_{2}}$ by $h_{1}, h_{2} \in \operatorname{Sp}(n, 1)$ to the geodesic $\gamma_{o \infty}$, we may assume that

$$
p_{1}=q_{1}=\mathbb{P}(\hat{\infty}+\hat{\mathbf{o}}), \quad p_{2}=q_{2}=\mathbb{P}\left(e^{\frac{t}{2}} \hat{\infty}+e^{-\frac{t}{2}} \hat{\mathbf{o}}\right)=\left(-e^{t}, 0, \cdots, 0\right),
$$

where $t=\rho\left(p_{1}, p_{2}\right)$.

Since

$$
\left\langle\hat{\infty}+\hat{\mathbf{o}}, e^{\frac{t}{2}} \hat{\infty}+e^{-\frac{t}{2}} \hat{\mathbf{o}}, \hat{\mathbf{p}}_{3}\right\rangle=\left(e^{\frac{t}{2}}+e^{-\frac{t}{2}}\right)\left(-e^{\frac{t}{2}}+e^{-\frac{t}{2}} r_{1}\right)\left(1-\overline{r_{1}}\right),
$$

the condition $\mathbb{A}_{\mathbb{H}}(\mathfrak{p})=\mathbb{A}_{\mathbb{H}}(\mathfrak{q})$ implies

$$
\frac{\Re\left[\left(r_{1}-e^{t}\right)\left(1-\overline{r_{1}}\right)\right]}{\left|\left(r_{1}-e^{t}\right)\left(1-\overline{r_{1}}\right)\right|}=\frac{\Re\left[\left(z_{1}-e^{t}\right)\left(1-\overline{z_{1}}\right)\right]}{\left|\left(z_{1}-e^{t}\right)\left(1-\overline{z_{1}}\right)\right|},
$$

that is

$$
\frac{\left(e^{t}+1\right) \Re\left(r_{1}\right)-\left|r_{1}\right|^{2}-e^{t}}{\left|\left(r_{1}-e^{t}\right)\left(1-\overline{r_{1}}\right)\right|}=\frac{\left(e^{t}+1\right) \Re\left(z_{1}\right)-\left|z_{1}\right|^{2}-e^{t}}{\left|\left(z_{1}-e^{t}\right)\left(1-\overline{z_{1}}\right)\right|} .
$$

It follows from $\rho\left(p_{1}, p_{3}\right)=\rho\left(q_{1}, q_{3}\right)$ that

$$
\frac{\left|r_{1}-1\right|^{2}}{2 \Re\left(r_{1}\right)+\sum_{i=2}^{n}\left|r_{i}\right|^{2}}=\frac{\left|z_{1}-1\right|^{2}}{2 \Re\left(z_{1}\right)+\sum_{i=2}^{n}\left|z_{i}\right|^{2}} .
$$


Similarly, $\rho\left(p_{2}, p_{3}\right)=\rho\left(q_{2}, q_{3}\right)$ implies that

$$
\frac{\left|r_{1}-e^{t}\right|^{2}}{2 \Re\left(r_{1}\right)+\sum_{i=2}^{n}\left|r_{i}\right|^{2}}=\frac{\left|z_{1}-e^{t}\right|^{2}}{2 \Re\left(z_{1}\right)+\sum_{i=2}^{n}\left|z_{i}\right|^{2}} .
$$

From equations (47)-(49) we obtain that

$$
\frac{\left(e^{t}+1\right) \Re\left(r_{1}\right)-\left|r_{1}\right|^{2}-e^{t}}{\left|1-r_{1}\right|^{2}}=\frac{\left(e^{t}+1\right) \Re\left(z_{1}\right)-\left|z_{1}\right|^{2}-e^{t}}{\left|1-z_{1}\right|^{2}} .
$$

It follows from the above formula that

$$
\frac{2 \Re\left(r_{1}\right)-1}{\left|r_{1}\right|^{2}}=\frac{2 \Re\left(z_{1}\right)-1}{\left|z_{1}\right|^{2}}
$$

By (48) and (49) we obtain that

$$
\frac{\left|r_{1}-1\right|^{2}}{\left|r_{1}-e^{t}\right|^{2}}=\frac{\left|z_{1}-1\right|^{2}}{\left|z_{1}-e^{t}\right|^{2}}
$$

We can rephrase the above equation as

$$
2\left|z_{1}\right|^{2} \Re\left(r_{1}\right)-\left(e^{t}+1\right)\left|z_{1}\right|^{2}+2 e^{t} \Re\left(z_{1}\right)=2\left|r_{1}\right|^{2} \Re\left(z_{1}\right)-\left(e^{t}+1\right)\left|r_{1}\right|^{2}+2 e^{t} \Re\left(r_{1}\right) .
$$

Substituting (51) into (52) we obtain

$$
\left|r_{1}\right|^{2}-2 \Re\left(r_{1}\right)=\left|z_{1}\right|^{2}-2 \Re\left(z_{1}\right) .
$$

Thus

$$
\left|r_{1}\right|^{2}\left(1-\frac{2 \Re\left(r_{1}\right)-1}{\left|r_{1}\right|^{2}}\right)=\left|z_{1}\right|^{2}\left(1-\frac{2 \Re\left(z_{1}\right)-1}{\left|z_{1}\right|^{2}}\right) .
$$

This implies $\left|z_{1}\right|=\left|r_{1}\right|$ and therefore $\Re\left(r_{1}\right)=\Re\left(z_{1}\right), \quad \sum_{i=2}^{n}\left|z_{i}\right|^{2}=\sum_{i=2}^{n}\left|r_{i}\right|^{2}$. Lemma 4.1 concludes the proof of Case (iv).

Remark 4.3. In Case (iv), since $p_{1}$ and $p_{2}$ lie in the geodesic $\gamma_{o \infty}$, we have $L_{o \infty}=L_{p_{1} p_{2}}$. It follows from $\rho\left(L_{o \infty}, r\right)=\rho\left(L_{o \infty}, q_{3}\right)$ that

$$
\frac{\Re\left(r_{1}\right)}{2 \Re\left(r_{1}\right)+\sum_{i=2}^{n}\left|r_{i}\right|^{2}}=\frac{\Re\left(z_{1}\right)}{2 \Re\left(z_{1}\right)+\sum_{i=2}^{n}\left|z_{i}\right|^{2}} .
$$

The equations (48), (49) and (55) imply that the condition (38) holds. This observation implies that we can replace the condition $\mathbb{A}_{\mathbb{H}}(\mathfrak{p})=\mathbb{A}_{\mathbb{H}}(\mathfrak{q})$ by $\rho\left(L_{p_{1} p_{3}}, p_{2}\right)=\rho\left(L_{q_{1} q_{3}}, q_{2}\right)$ in Case (iv) of Theorem 1.1 .

\section{The moduli space of quadruples of pairwise distinct points of $\partial \mathbf{H}_{\mathbb{H}}^{n}$}

\subsection{The Gram matrix}

Given a quadruple $\mathfrak{p}=\left(p_{1}, p_{2}, p_{3}, p_{4}\right)$ in $\partial \mathbf{H}_{\mathbb{H}}^{n}$ with lift $\mathbf{p}=\left(\mathbf{p}_{1}, \mathbf{p}_{2}, \mathbf{p}_{3}, \mathbf{p}_{4}\right)$. The following Hermitian matrix

$$
G=G(\mathbf{p})=\left(g_{i j}\right)=\left(\left\langle\mathbf{p}_{i}, \mathbf{p}_{j}\right\rangle\right)
$$

is called the Gram matrix associated to $\mathfrak{p}$. It is obvious that

$$
G(\mathbf{p})=G(f \mathbf{p})=G\left(f \mathbf{p}_{1}, f \mathbf{p}_{2}, f \mathbf{p}_{3}, f \mathbf{p}_{4}\right), f \in \operatorname{PSp}(n, 1) .
$$


Let $D=\operatorname{diag}\left(\lambda_{1}, \lambda_{2}, \lambda_{3}, \lambda_{4}\right)$ and $\tilde{\mathbf{p}}=\left(\mathbf{p}_{1} \lambda_{1}, \mathbf{p}_{2} \lambda_{2}, \mathbf{p}_{3} \lambda_{3}, \mathbf{p}_{4} \lambda_{4}\right)$. Then

$$
\tilde{G}=G(\tilde{\mathbf{p}})=\left(\left\langle\mathbf{p}_{i} \lambda_{i}, \mathbf{p}_{j} \lambda_{j}\right\rangle\right)=\left(\overline{\lambda_{j}}\left\langle\mathbf{p}_{i}, \mathbf{p}_{j}\right\rangle \lambda_{i}\right)
$$

and

$$
\overline{\tilde{G}}=D^{*} \bar{G} D
$$

where $\bar{G}=\left(\overline{g_{i j}}\right)$ is the conjugate of $G$.

We say that two Hermitian matrices $H$ and $\tilde{H}$ are equivalent if there exists a diagonal matrix

$$
D=\operatorname{diag}\left(\lambda_{1}, \lambda_{2}, \lambda_{3}, \lambda_{4}\right), \lambda_{i} \in \mathbb{H} \backslash\{0\}
$$

such that

$$
\tilde{H}=D^{*} H D \text {. }
$$

Thus, to each quadruple $\mathbf{p}$ of points in $V_{0}$ is associated an equivalence class of Hermitian matrices with zeros on the diagonal.

Proposition 5.1. Let $\mathfrak{p}=\left(p_{1}, p_{2}, p_{3}, p_{4}\right)$ be a quadruple of pairwise distinct points in $\partial \mathbf{H}_{\mathbb{H}}^{n}$. Then the equivalence class of Gram matrices associated to $\mathfrak{p}$ contains a unique matrix $G=\left(g_{i j}\right)$ with

$$
g_{i i}=0, i=1, \cdots 4, g_{12}=g_{23}=g_{34}=1 \text {, }
$$

and

$$
g_{13}=-e^{\mathbf{i} \mathbb{A}}, g_{14}=c_{1}+t \mathbf{j}, g_{24}=c_{2}+c_{3} \mathbf{j},
$$

where $\mathbb{A}=\mathbb{A}_{\mathbb{H}}\left(\left(p_{1}, p_{2}, p_{3}\right)\right), t \geq 0$ and $c_{i} \in \mathbb{C}, i=1,2,3$. Furthermore $c_{3} \geq 0$ provided $t=0$.

Proof. Let $\mathbf{p}=\left(\mathbf{p}_{1}, \mathbf{p}_{2}, \mathbf{p}_{3}, \mathbf{p}_{4}\right)$ be an arbitrary lift of $\mathfrak{p}$. We want to obtain a lift $\mathbf{n}$ of $\mathfrak{p}$ by rescaling $\mathbf{p}$ such that $G(\mathbf{n})$ is the desired Gram matrix.

Note that $\left\langle\mathbf{p}_{i}, \mathbf{p}_{j}\right\rangle \neq 0$ for $i \neq j$. Firstly we obtain $\lambda_{i}, i=2,3,4$ as the solutions of the following equations:

$$
\left\langle\mathbf{p}_{1}, \mathbf{p}_{2} \lambda_{2}\right\rangle=1,\left\langle\mathbf{p}_{2} \lambda_{2}, \mathbf{p}_{3} \lambda_{3}\right\rangle=1,\left\langle\mathbf{p}_{3} \lambda_{3}, \mathbf{p}_{4} \lambda_{4}\right\rangle=1 \text {. }
$$

These solutions $\lambda_{i}, i=2,3,4$ are given by (10) in terms of $\mathbf{p}_{i}, i=1,2,3,4$. Now the Gram matrix $G$ of $\left(\mathbf{p}_{1}, \mathbf{p}_{2} \lambda_{2}, \mathbf{p}_{3} \lambda_{3}, \mathbf{p}_{4} \lambda_{4}\right)$ satisfies $g_{i i}=0, i=1, \cdots 4, g_{12}=g_{23}=g_{34}=1$.

Secondly, we want to find $\lambda_{1}$ such that the Gram matrix $G=\left(g_{i j}\right)$ of

$$
\left(\mathbf{p}_{1} \lambda_{1}, \mathbf{p}_{2} \lambda_{2}{\overline{\lambda_{1}}}^{-1}, \mathbf{p}_{3} \lambda_{3} \lambda_{1}, \mathbf{p}_{4} \lambda_{4}{\overline{\lambda_{1}}}^{-1}\right)
$$

satisfies $g_{i i}=0, i=1, \cdots 4, g_{12}=g_{23}=g_{34}=1, g_{13}=-e^{\mathbf{i}}$. We mention that the requirement

$$
g_{13}=\left\langle\mathbf{p}_{1} \lambda_{1}, \mathbf{p}_{3} \lambda_{3} \lambda_{1}\right\rangle=-e^{\mathbf{i} \mathbb{A}}
$$

comes from Proposition 1.2 and the property (29). We can verify that $\lambda_{1}$ of the form (11) is the desired solution.

We mention that $\lambda_{1}$ given by (11) is just a specific choice. For example, let $\lambda_{1}^{\prime}=\lambda_{1} e^{\mathbf{i} \theta}$ for arbitrary $\theta$ and $\lambda_{i}^{\prime}=\lambda_{i}, i=2,3,4$. Then the Gram matrix $G^{\prime}=\left(g_{i j}^{\prime}\right)$ of $\left(\mathbf{p}_{1} \lambda_{1}^{\prime}, \mathbf{p}_{2} \lambda_{2}^{\prime}{\overline{\lambda_{1}^{\prime}}}^{-1}, \mathbf{p}_{3} \lambda_{3}^{\prime} \lambda_{1}^{\prime}, \mathbf{p}_{4} \lambda_{4}^{\prime}{\overline{\lambda_{1}^{\prime}}}^{-1}\right)$ also satisfies $g_{i i}^{\prime}=0, i=1, \cdots 4, g_{12}^{\prime}=g_{23}^{\prime}=g_{34}^{\prime}=1, g_{13}^{\prime}=-e^{\mathrm{i} \mathbb{A}}$. However

$$
g_{14}^{\prime}=e^{-\mathbf{i} \theta} g_{14} e^{\mathbf{i} \theta}, \quad g_{24}^{\prime}=e^{-\mathbf{i} \theta} g_{24} e^{\mathbf{i} \theta} .
$$

It is the function $\sigma(a, b)$ which eliminates this indeterminacy in the following third step. 
Thirdly we set

$$
\mu=\sigma\left(\left\langle\mathbf{p}_{1} \lambda_{1}, \mathbf{p}_{4} \lambda_{4}{\overline{\lambda_{1}}}^{-1}\right\rangle,\left\langle\mathbf{p}_{2} \lambda_{2}{\overline{\lambda_{1}}}^{-1}, \mathbf{p}_{4} \lambda_{4}{\overline{\lambda_{1}}}^{-1}\right\rangle\right) .
$$

Then the Gram matrix of lift

$$
\mathbf{n}=\left(\mathbf{n}_{1}, \mathbf{n}_{2}, \mathbf{n}_{3}, \mathbf{n}_{4}\right)=\left(\mathbf{p}_{1} \lambda_{1} \mu, \mathbf{p}_{2} \lambda_{2}{\overline{\lambda_{1}}}^{-1} \mu, \mathbf{p}_{3} \lambda_{3} \lambda_{1} \mu, \mathbf{p}_{4} \lambda_{4}{\overline{\lambda_{1}}}^{-1} \mu\right)
$$

is the desired Gram matrix.

The matrix $G$ as in Proposition 5.1 is called the normalised Gram matrix, which is denoted by $G(\mathfrak{p})$. The corresponding lift $\mathbf{n}=\left(\mathbf{n}_{1}, \mathbf{n}_{2}, \mathbf{n}_{3}, \mathbf{n}_{4}\right)$ given by (59) of $\mathfrak{p}$ is called the normalised lift. That is

$$
G(\mathfrak{p})=G(\mathbf{n})=\left(g_{i j}\right)=\left(\begin{array}{cccc}
0 & 1 & -e^{\mathbf{i} \mathbb{A}} & \kappa_{1} \\
1 & 0 & 1 & \kappa_{2} \\
-e^{-i \mathbb{A}} & 1 & 0 & 1 \\
\overline{\kappa_{1}} & \overline{\kappa_{2}} & 1 & 0
\end{array}\right)
$$

where $\kappa_{1}=c_{1}+t \mathbf{j}$ and $\kappa_{2}=c_{2}+c_{3} \mathbf{j}$.

Let $\mathbf{p}=\left(\mathbf{p}_{1}, \mathbf{p}_{2}, \mathbf{p}_{3}, \mathbf{p}_{4}\right)$ be a quadruple of points in $V_{0} \cup V_{-}$such that $\mathbb{P}(\mathbf{p})$ are pairwise distinct points in $\overline{\mathbf{H}_{\mathbb{H}}^{n}}$. We define the following three cross-ratios:

$$
\mathbb{X}_{1}(\mathbf{p})=\mathbb{X}\left(\mathbf{p}_{1}, \mathbf{p}_{2}, \mathbf{p}_{3}, \mathbf{p}_{4}\right), \mathbb{X}_{2}(\mathbf{p})=\mathbb{X}\left(\mathbf{p}_{2}, \mathbf{p}_{4}, \mathbf{p}_{3}, \mathbf{p}_{1}\right), X_{3}(\mathbf{p})=\mathbb{X}\left(\mathbf{p}_{1}, \mathbf{p}_{4}, \mathbf{p}_{3}, \mathbf{p}_{2}\right) .
$$

Proposition 5.2. Let $G(\mathfrak{p})$ be a normalised Gram matrix given by (60) with a normalised lift

$$
\mathbf{n}=\left(\mathbf{n}_{1}, \mathbf{n}_{2}, \mathbf{n}_{3}, \mathbf{n}_{4}\right)=\left(\mathbf{p}_{1} \nu_{1}, \mathbf{p}_{2} \nu_{2}, \mathbf{p}_{3} \nu_{3}, \mathbf{p}_{4} \nu_{4}\right)
$$

given by (59). That is

$$
\nu_{1}=\lambda_{1} \mu, \nu_{2}=\lambda_{2}{\overline{\lambda_{1}}}^{-1} \mu, \nu_{3}=\lambda_{3} \lambda_{1} \mu, \nu_{4}=\lambda_{4}{\overline{\lambda_{1}}}^{-1} \mu
$$

Then

(i) $\mathbb{X}_{1}(\mathbf{n})=-e^{-\mathbf{i} \mathbb{A}} \overline{\kappa_{2}} \overline{\kappa_{1}}-1, \mathbb{X}_{2}(\mathbf{n})=\kappa_{1}, \quad \mathbb{A}_{\mathbb{H}}\left(\mathbf{n}_{1}, \mathbf{n}_{2}, \mathbf{n}_{3}\right)=\mathbb{A} ;$

(ii) $\kappa_{1}=\mathbb{X}_{2}(\mathbf{n}), \kappa_{2}=-\mathbb{X}_{2}(\mathbf{n}) \overline{\mathbb{X}_{1}(\mathbf{n})} e^{-\mathbf{i} \mathbb{A}}, \mathbb{A}=\mathbb{A}_{\mathbb{H}}\left(\mathbf{n}_{1}, \mathbf{n}_{2}, \mathbf{n}_{3}\right)$;

(iii) $\mathbb{X}_{1}(\mathbf{p})={\overline{\nu_{1}}}^{-1} \mathbb{X}_{1}(\mathbf{n}) \overline{\nu_{1}}, \mathbb{X}_{2}(\mathbf{p})={\overline{\nu_{2}}}^{-1} \mathbb{X}_{2}(\mathbf{n}) \overline{\nu_{2}}, \mathbb{A}_{\mathbb{H}}\left(\mathbf{p}_{1}, \mathbf{p}_{2}, \mathbf{p}_{3}\right)=\mathbb{A}_{\mathbb{H}}\left(\mathbf{n}_{1}, \mathbf{n}_{2}, \mathbf{n}_{3}\right) ;$

(iv) $\kappa_{1}=\overline{\nu_{2}} \mathbb{X}_{2}(\mathbf{p}){\overline{\nu_{2}}}^{-1}, \kappa_{2}=-\overline{\nu_{2}} \mathbb{X}_{2}(\mathbf{p}) \overline{\nu_{2}}-1 \nu_{1}^{-1} \overline{\mathbb{X}_{1}(\mathbf{p})} \nu_{1} e^{-\mathbf{i} \mathbb{A}_{\mathbb{H}}\left(\mathbf{p}_{1}, \mathbf{p}_{2}, \mathbf{p}_{3}\right)}, \mathbb{A}=\mathbb{A}_{\mathbb{H}}\left(\mathbf{p}_{1}, \mathbf{p}_{2}, \mathbf{p}_{3}\right) ;$

(v) $\mathbb{X}_{3}(\mathbf{p})={\overline{\nu_{1}}}^{-1} \mathbb{X}_{3}(\mathbf{n}) \overline{\nu_{1}}, \quad \mathbb{X}_{3}(\mathbf{n})=-e^{-\mathbf{i} \mathbb{A}} \kappa_{2}$.

Proof. Since $\left\langle\mathbf{n}_{1}, \mathbf{n}_{3}\right\rangle=-e^{\mathbf{i} \mathbb{A}},\left\langle\mathbf{n}_{1}, \mathbf{n}_{4}\right\rangle=\kappa_{1}$ and $\left\langle\mathbf{n}_{2}, \mathbf{n}_{4}\right\rangle=\kappa_{2}$, we have

$$
\begin{gathered}
\mathbb{X}_{1}(\mathbf{n})=\mathbb{X}\left(\mathbf{n}_{1}, \mathbf{n}_{2}, \mathbf{n}_{3}, \mathbf{n}_{4}\right)=\left\langle\mathbf{n}_{3}, \mathbf{n}_{1}\right\rangle\left\langle\mathbf{n}_{3}, \mathbf{n}_{2}\right\rangle^{-1}\left\langle\mathbf{n}_{4}, \mathbf{n}_{2}\right\rangle\left\langle\mathbf{n}_{4}, \mathbf{n}_{1}\right\rangle^{-1}=-e^{-\mathbf{i} \mathbb{A}} \overline{\kappa_{2}} \overline{\kappa_{1}}, \\
\mathbb{X}_{2}(\mathbf{n})=\left\langle\mathbf{n}_{3}, \mathbf{n}_{2}\right\rangle\left\langle\mathbf{n}_{3}, \mathbf{n}_{4}\right\rangle^{-1}\left\langle\mathbf{n}_{1}, \mathbf{n}_{4}\right\rangle\left\langle\mathbf{n}_{1}, \mathbf{n}_{2}\right\rangle^{-1}=\kappa_{1}
\end{gathered}
$$

and

$$
\mathbb{X}_{3}(\mathbf{n})=\left\langle\mathbf{n}_{3}, \mathbf{n}_{1}\right\rangle\left\langle\mathbf{n}_{3}, \mathbf{n}_{4}\right\rangle^{-1}\left\langle\mathbf{n}_{2}, \mathbf{n}_{4}\right\rangle\left\langle\mathbf{n}_{2}, \mathbf{n}_{1}\right\rangle^{-1}=-e^{-\mathbf{i} \mathbb{A}} \kappa_{2} .
$$

By properties of quaternionic Cartan's angular invariant, we have $\mathbb{A}_{\mathbb{H}}\left(\mathbf{n}_{1}, \mathbf{n}_{2}, \mathbf{n}_{3}\right)=\mathbb{A}_{\mathbb{H}}\left(\mathbf{p}_{1}, \mathbf{p}_{2}, \mathbf{p}_{3}\right)=\mathbb{A}$. Since $\mathbf{p}_{i}=\mathbf{n}_{i} \nu_{i}^{-1}$, by (21) we have

$$
\mathbb{X}_{1}(\mathbf{p})=\mathbb{X}\left(\mathbf{n}_{1} \nu_{1}^{-1}, \mathbf{n}_{2} \nu_{2}^{-1}, \mathbf{n}_{3} \nu_{3}^{-1}, \mathbf{n}_{4} \nu_{4}^{-1}\right)=\overline{\nu_{1}^{-1}} \mathbb{X}_{1}(\mathbf{n}) \overline{\nu_{1}}
$$

Similarly, we obtain $\mathbb{X}_{2}(\mathbf{p})={\overline{\nu_{2}}}^{-1} \mathbb{X}_{2}(\mathbf{n}) \overline{\nu_{2}}, \quad \mathbb{X}_{3}(\mathbf{p})={\overline{\nu_{1}}}^{-1} \mathbb{X}_{3}(\mathbf{n}) \overline{\nu_{1}}$. Therefore (i)-(v) hold. 
Proposition 5.3. Let $\kappa_{1}, \kappa_{2}, \mathbb{A}$ be given by (9) stemming from $\mathbf{p}=\left(\mathbf{p}_{1}, \mathbf{p}_{2}, \mathbf{p}_{3}, \mathbf{p}_{4}\right)$. Then

(i) $\Re\left(\kappa_{2}\right)=-\left|\kappa_{2}\right| \cos \mathbb{A}_{\mathbb{H}}\left(\mathbf{p}_{2}, \mathbf{p}_{3}, \mathbf{p}_{4}\right) \leq 0$;

(ii) $\cos \mathbb{A}=\cos \mathbb{A}_{\mathbb{H}}\left(\mathbf{p}_{1}, \mathbf{p}_{2}, \mathbf{p}_{3}\right) \geq 0$;

(iii) $\Re\left(\kappa_{1} \overline{\kappa_{2}}\right)=-\left|\kappa_{1}\right|\left|\kappa_{2}\right| \cos \mathbb{A}_{\mathbb{H}}\left(\mathbf{p}_{1}, \mathbf{p}_{2}, \mathbf{p}_{4}\right) \leq 0$;

(iv)

$$
\left|\kappa_{1}\right|=\left|\mathbb{X}_{2}(\mathbf{p})\right|=\frac{\left|\left\langle\mathbf{p}_{1}, \mathbf{p}_{4}\right\rangle\right|\left|\left\langle\mathbf{p}_{2}, \mathbf{p}_{3}\right\rangle\right|}{\left|\left\langle\mathbf{p}_{1}, \mathbf{p}_{2}\right\rangle\right|\left|\left\langle\mathbf{p}_{3}, \mathbf{p}_{4}\right\rangle\right|},\left|\kappa_{2}\right|=\left|\mathbb{X}_{3}(\mathbf{p})\right|=\left|\mathbb{X}_{1}(\mathbf{p})\right|\left|\mathbb{X}_{2}(\mathbf{p})\right|=\frac{\left|\left\langle\mathbf{p}_{1}, \mathbf{p}_{3}\right\rangle\right|\left|\left\langle\mathbf{p}_{2}, \mathbf{p}_{4}\right\rangle\right|}{\left|\left\langle\mathbf{p}_{1}, \mathbf{p}_{2}\right\rangle\right|\left|\left\langle\mathbf{p}_{3}, \mathbf{p}_{4}\right\rangle\right|}
$$

Proof. Since $\kappa_{2}=\left\langle\mathbf{n}_{2}, \mathbf{n}_{4}\right\rangle=\left\langle\mathbf{n}_{2}, \mathbf{n}_{3}, \mathbf{n}_{4}\right\rangle$, by Proposition 1.2 we have

$$
\Re\left(-\kappa_{2}\right)=\Re\left(-\left\langle\mathbf{n}_{2}, \mathbf{n}_{3}, \mathbf{n}_{4}\right\rangle\right)=\left|\kappa_{2}\right| \frac{\Re\left(-\left\langle\mathbf{n}_{2}, \mathbf{n}_{3}, \mathbf{n}_{4}\right\rangle\right)}{\left|\left\langle\mathbf{n}_{2}, \mathbf{n}_{3}, \mathbf{n}_{4}\right\rangle\right|}=\left|\kappa_{2}\right| \cos \mathbb{A}_{\mathbb{H}}\left(\mathbf{p}_{2}, \mathbf{p}_{3}, \mathbf{p}_{4}\right) \geq 0 .
$$

It follows from $e^{\mathbf{i} \mathbb{A}}=-\left\langle\mathbf{n}_{1}, \mathbf{n}_{3}\right\rangle=-\left\langle\mathbf{n}_{1}, \mathbf{n}_{2}, \mathbf{n}_{3}\right\rangle$ that

$$
\cos \mathbb{A}=\Re\left(-\left\langle\mathbf{n}_{1}, \mathbf{n}_{2}, \mathbf{n}_{3}\right\rangle\right)=\frac{\Re\left(-\left\langle\mathbf{n}_{1}, \mathbf{n}_{2}, \mathbf{n}_{3}\right\rangle\right)}{\left|\left\langle\mathbf{n}_{1}, \mathbf{n}_{2}, \mathbf{n}_{3}\right\rangle\right|}=\cos \mathbb{A}_{\mathbb{H}}\left(\mathbf{n}_{1}, \mathbf{n}_{2}, \mathbf{n}_{3}\right)=\cos \mathbb{A}_{\mathbb{H}}\left(\mathbf{p}_{1}, \mathbf{p}_{2}, \mathbf{p}_{3}\right) \geq 0 .
$$

Noting that $\kappa_{1}=\left\langle\mathbf{n}_{1}, \mathbf{n}_{4}\right\rangle, \kappa_{2}=\left\langle\mathbf{n}_{2}, \mathbf{n}_{4}\right\rangle$ and $\left\langle\mathbf{n}_{2}, \mathbf{n}_{1}\right\rangle=1$, we have

$$
\begin{aligned}
\Re\left(\kappa_{1} \overline{\kappa_{2}}\right) & =\Re\left(\overline{\kappa_{2}} \kappa_{1}\right)=\Re\left(\left\langle\mathbf{n}_{4}, \mathbf{n}_{2}\right\rangle\left\langle\mathbf{n}_{1}, \mathbf{n}_{4}\right\rangle\right)=\Re\left(\left\langle\mathbf{n}_{2}, \mathbf{n}_{1}\right\rangle\left\langle\mathbf{n}_{4}, \mathbf{n}_{2}\right\rangle\left\langle\mathbf{n}_{1}, \mathbf{n}_{4}\right\rangle\right) \\
& =-\Re\left(-\left\langle\mathbf{n}_{1}, \mathbf{n}_{2}, \mathbf{n}_{4}\right\rangle\right)=-\left|\kappa_{1}\right|\left|\kappa_{2}\right| \cos \mathbb{A}_{\mathbb{H}}\left(\mathbf{n}_{1}, \mathbf{n}_{2}, \mathbf{n}_{4}\right)=-\left|\kappa_{1}\right|\left|\kappa_{2}\right| \cos \mathbb{A}_{\mathbb{H}}\left(\mathbf{p}_{1}, \mathbf{p}_{2}, \mathbf{p}_{4}\right) \leq 0 .
\end{aligned}
$$

Therefore we prove (i)-(iii). The assertion (iv) follows from (ii) and (v) of Proposition 5.2

We remark that one can find more relationship of quaternionic cross-ratios in Sections 3, 4 of [20].

\subsection{The moduli space}

We know from Proposition 5.3 that a Hermitian matrix of the form (60) need satisfy some conditions to be a normalised Gram matrix. These conditions are described in the following theorem.

Theorem 5.1. Let $G$ be a matrix of the form (60) with

$$
\mathbb{A} \in[0, \pi / 2], \quad \Re\left(\kappa_{1} \overline{\kappa_{2}}\right) \leq 0, \Re\left(\kappa_{2}\right) \leq 0, \kappa_{1} \neq 0, \quad \kappa_{2} \neq 0 .
$$

Denote by

$$
D(G)=1+\left|\kappa_{1}\right|^{2}+\left|\kappa_{2}\right|^{2}-2 \Re\left(\kappa_{1}\right)+2 \Re\left(\kappa_{2} e^{-\mathbf{i} \mathbb{A}}\right)+2 \Re\left(\overline{\kappa_{1}} \kappa_{2} e^{\mathbf{i} \mathbb{A}}\right) .
$$

Then we have the followings.

(i) When $n=2, G$ is the normalised Gram matrix for some quadruple of pairwise distinct points in $\partial \mathbf{H}_{\mathbb{H}}^{n}$ if and only if

$$
D(G)=0
$$

(ii) When $n>2, G$ is the normalised Gram matrix for some quadruple of pairwise distinct points in $\partial \mathbf{H}_{\mathbb{H}}^{n}$ if and only if

$$
D(G) \leq 0
$$

Moreover, $D(G)=0$ if and only if there exist $\lambda_{i} \in \mathbb{H}$ with $\sum_{i=1}^{4}\left|\lambda_{i}\right| \neq 0$ such that $\sum_{i=1}^{4} \mathbf{n}_{i} \lambda_{i}=0$. 
Proof. We first prove necessity. Suppose that $G(\mathbf{n})$ is the normalised matrix with a normalised lift $\mathbf{n}=\left(\mathbf{n}_{1}, \mathbf{n}_{2}, \mathbf{n}_{3}, \mathbf{n}_{4}\right)$. Since $G(\mathfrak{p})=G(\mathbf{n})=G(f \mathbf{n})$, we have freedom to choose some specific normalised lifts. Since $\operatorname{PSp}(n, 1)$ acts doubly transitively on $V_{0}$, by our normalised process we may assume that

$$
\mathbf{n}_{1}=\left(\begin{array}{c}
0 \\
\vdots \\
0 \\
\lambda
\end{array}\right), \quad \mathbf{n}_{2}=\left(\begin{array}{c}
\bar{\lambda}^{-1} \\
0 \\
\vdots \\
0
\end{array}\right), \quad \mathbf{n}_{3}=\left(\begin{array}{c}
-\bar{\lambda}^{-1} e^{-\mathbf{i} \mathbb{A}} \\
\alpha \\
\lambda
\end{array}\right), \quad \mathbf{n}_{4}=\left(\begin{array}{c}
\bar{\lambda}^{-1} \overline{\kappa_{1}} \\
\beta \\
\lambda \overline{\kappa_{2}}
\end{array}\right),
$$

where $\alpha, \beta$ are column vectors in $\mathbb{H}^{n-1}$. By $\left\langle\mathbf{n}_{3}, \mathbf{n}_{4}\right\rangle=1$ and $\left\langle\mathbf{n}_{3}, \mathbf{n}_{3}\right\rangle=\left\langle\mathbf{n}_{4}, \mathbf{n}_{4}\right\rangle=0$, we have

$$
\beta^{*} \alpha=1+\kappa_{2} e^{-\mathbf{i} \mathbb{A}}-\kappa_{1},|\alpha|^{2}=2 \cos \mathbb{A},|\beta|^{2}=-2 \Re\left(\kappa_{1} \overline{\kappa_{2}}\right) .
$$

Note that

$$
|\beta|^{2}|\alpha|^{2} \geq\left|\beta^{*} \alpha\right|^{2}
$$

That is

$$
\begin{aligned}
-4 \cos \mathbb{A} \Re\left(\kappa_{1} \overline{\kappa_{2}}\right) & \geq\left|1+\kappa_{2} e^{-\mathbf{i} \mathbb{A}}-\kappa_{1}\right|^{2} \\
& =1+\left|\kappa_{1}\right|^{2}+\left|\kappa_{2}\right|^{2}-2 \Re\left(\kappa_{1}\right)+2 \Re\left(\kappa_{2} e^{-\mathbf{i} \mathbb{A}}\right)-2 \Re\left(\overline{\kappa_{2}} \kappa_{1} e^{\mathbf{i} \mathbb{A}}\right) \\
& =D(G)-2 \Re\left(\overline{\kappa_{1}} \kappa_{2} e^{\mathbf{i} \mathbb{A}}\right)-2 \Re\left(\overline{\kappa_{2}} \kappa_{1} e^{\mathbf{i} \mathbb{A}}\right) \\
& =D(G)-2\left(\overline{\kappa_{1}} \kappa_{2}+\overline{\kappa_{2}} \kappa_{1}\right) \Re\left(e^{\mathbf{i} \mathbb{A}}\right) .
\end{aligned}
$$

Therefore

$$
D(G)=1+\left|\kappa_{1}\right|^{2}+\left|\kappa_{2}\right|^{2}-2 \Re\left(\kappa_{1}\right)+2 \Re\left(\kappa_{2} e^{-\mathbf{i} \mathbb{A}}\right)+2 \Re\left(\overline{\kappa_{1}} \kappa_{2} e^{\mathbf{i} \mathbb{A}}\right) \leq 0 .
$$

$D(G)=0$ if and only if $\beta=\alpha \mu$ or $\alpha=\beta \nu$ for some $\mu, \nu \in \mathbb{H}$. Therefore the case $D(G)=0$ implies that there exist $\lambda_{i} \in \mathbb{H}$ with $\sum_{i=1}^{4}\left|\lambda_{i}\right| \neq 0$ such that $\sum_{i=1}^{4} \mathbf{n}_{i} \lambda_{i}=0 . \quad \alpha$ and $\beta$ are quaternions for the case $n=2$, which implies that we always have $D(G)=0$ for this case.

For sufficiency, we need to find an $\mathbf{n}=\left(\mathbf{n}_{1}, \mathbf{n}_{2}, \mathbf{n}_{3}, \mathbf{n}_{4}\right)$ whose normalised Gram matrix is $G$ under the conditions (63) and (65), or (66). We consider the normalised polar vectors of the following form:

$$
\mathbf{n}_{1}=(0, \cdots, 0, \lambda)^{T}, \mathbf{n}_{2}=\left(\bar{\lambda}^{-1}, 0, \cdots, 0\right)^{T}, \mathbf{n}_{3}=\left(-\bar{\lambda}^{-1} e^{-\mathbf{i} \mathbb{A}}, \alpha^{T}, \lambda\right)^{T}, \mathbf{n}_{4}=\left(\bar{\lambda}^{-1} \overline{\kappa_{1}}, \beta^{T}, \lambda \overline{\kappa_{2}}\right)^{T},
$$

where $\lambda \in \mathbb{H}, \alpha, \beta \in \mathbb{H}^{n-1}$. We need to find solutions of the following underdetermined system of equations:

$$
\begin{aligned}
\beta^{*} \alpha & =1+\kappa_{2} e^{-\mathbf{i} \mathbb{A}}-\kappa_{1}, \\
|\alpha|^{2} & =2 \cos \mathbb{A}, \\
|\beta|^{2} & =-2 \Re\left(\kappa_{1} \overline{\kappa_{2}}\right) .
\end{aligned}
$$

We first consider the case $D(G)=0$.

Note that

$$
D(G)=\left|1+\kappa_{2} e^{-\mathbf{i} \mathbb{A}}-\kappa_{1}\right|^{2}+4 \cos \mathbb{A} \Re\left(\kappa_{1} \overline{\kappa_{2}}\right) .
$$

If $\mathbb{A}=\pi / 2$ then

$$
\lambda=1, \alpha=(0, \cdots, 0)^{T}, \beta=\left(\sqrt{-2 \Re\left(\kappa_{1} \overline{\kappa_{2}}\right)}, 0, \cdots, 0\right)^{T} .
$$

are the desired solutions. If $\mathbb{A} \neq \pi / 2$ then

$$
\lambda=1, \alpha=(\sqrt{2 \cos \mathbb{A}}, 0, \cdots, 0)^{T}, \beta=\alpha \mu, \text { where } \mu=\frac{1+e^{\mathbf{i} \mathbb{A}} \overline{\kappa_{2}}-\overline{\kappa_{1}}}{2 \cos \mathbb{A}},
$$


are the desired solutions.

For the case $D(G)<0$ and $n>2$,

$$
\lambda=1, \alpha=(\sqrt{2 \cos \mathbb{A}}, 0, \cdots, 0)^{T}, \beta=\left(\frac{1+e^{\mathrm{i} \mathbb{A}} \overline{\kappa_{2}}-\overline{\kappa_{1}}}{\sqrt{2 \cos \mathbb{A}}}, 0, \cdots, 0, \frac{\sqrt{-D(G)}}{\sqrt{2 \cos \mathbb{A}}}\right)^{T} .
$$

are the desired solutions.

We mention that (63) and (64) can be rephrased in terms of the parameters $\left(c_{1}, c_{2}, c_{3}, t ; \mathbb{A}\right)$ as

$$
\mathbb{A} \in[0, \pi / 2], \quad \Re\left(c_{1} \overline{c_{2}}\right)+t \Re\left(c_{3}\right) \leq 0, \Re\left(c_{2}\right) \leq 0, \quad t \geq 0, \quad\left|c_{1}\right|^{2}+t^{2} \neq 0, \quad\left|c_{2}\right|^{2}+\left|c_{3}\right|^{2} \neq 0
$$

and

$$
D(G)=1+\left|c_{1}\right|^{2}+\left|c_{2}\right|^{2}+\left|c_{3}\right|^{2}+t^{2}-2 \Re\left(c_{1}\right)+2 \Re\left(c_{2} e^{-\mathbf{i} \mathbb{A}}\right)+2 \Re\left(\left(\overline{c_{1}} c_{2}+t \overline{c_{3}}\right) e^{\mathbf{i} \mathbb{A}}\right) .
$$

Remark 5.1. In the complex case, we can view $\mathbf{n}=\left(\mathbf{n}_{1}, \mathbf{n}_{2}, \mathbf{n}_{3}, \mathbf{n}_{4}\right)$ as a matrix. Then the normalised matrix $G$ is

$$
G=\mathbf{n}^{*} J \mathbf{n}
$$

Therefore

$$
\operatorname{det} G=|\operatorname{det} \mathbf{n}|^{2} \operatorname{det} J=-|\operatorname{det} \mathbf{n}|^{2} \leq 0 .
$$

$\operatorname{det} G=0$ if and only if $\operatorname{det} \mathbf{n}=0$. The case $\operatorname{det} \mathbf{n}=0$ implies that $\mathbf{n}_{1}, \mathbf{n}_{2}, \mathbf{n}_{3}$ and $\mathbf{n}_{4}$ are linearly dependent.

We now prove Theorem 1.2 ,

Proof of Theorem 1.2. By our normalised process in Proposition 5.1, the map $\tau$ define a map $\tau: \mathcal{M}(n) \rightarrow \mathbb{M}(n)$. Theorem 5.1 implies that such map is bijective. It is obvious that $\tau: \mathcal{M}(n) \rightarrow \mathbb{M}(n)$ is a homeomorphism because $\mathbb{M}(n)$ has the topology structure induced from $\mathbb{C}^{3} \times \mathbb{R} \times \mathbb{R}$.

\subsection{The congruence class of quadruple of pairwise distinct points of $\partial \mathbf{H}_{\mathbb{H}}^{n}$}

Similarly to Proposition 2.2 and Corollary 2.3 in [9], we can prove the following proposition.

Proposition 5.4. Let $\mathfrak{p}=\left(p_{1}, p_{2}, p_{3}, p_{4}\right)$ and $\mathfrak{q}=\left(q_{1}, q_{2}, q_{3}, q_{4}\right)$ be two quadruples of pairwise distinct points in $\partial \mathbf{H}_{\mathbb{H}}^{n}$. Then

(i) $\mathfrak{p}$ and $\mathfrak{q}$ are congruent in $\operatorname{PSp}(n, 1)$ if and only if their associated Gram matrices are equivalent.

(ii) $\mathfrak{p}$ and $\mathfrak{q}$ are congruent in $\operatorname{PSp}(n, 1)$ if and only if $G(\mathfrak{p})=G(\mathfrak{q})$.

We theoretically solve the problem whether two quadruples of pairwise distinct points in $\partial \mathbf{H}_{\mathbb{H}}^{n}$ are in the same congruence class or not by Proposition 5.4(ii). We can figure out the corresponding parameters $\left(c_{1}, c_{2}, c_{3}, t ; \mathbb{A}\right)$ by the three steps in Section 1 and compare them. When one consider this problem only using the geometric invariants such as the involved quaternionic cross-ratios and quaternionic Cartan's angular invariants, Propositions 5.2 and 5.3 also provide some equalities to compute the parameters $\left(c_{1}, c_{2}, c_{3}, t ; \mathbb{A}\right)$. However, we have used $\nu_{1}$ and $\nu_{2}$ to describe the relationship between $\mathbb{X}_{i}(\mathbf{p})$ and $\mathbb{X}_{i}(\mathbf{n}), i=$ 1,2 in Proposition 5.2 (ii). Note that $\nu_{1}$ and $\nu_{2}$ are expressed by the two functions $\nu(a)$ and $\sigma(a, b)$. Therefore it is difficult for us to obtain the parameters $\left(c_{1}, c_{2}, c_{3}, t ; \mathbb{A}\right)$ in terms of $\mathbb{X}_{i}(\mathbf{p}), i=1,2,3$ and $\mathbb{A}_{\mathbb{H}}\left(\mathbf{p}_{\iota(1)}, \mathbf{p}_{\iota(2)}, \mathbf{p}_{\iota(3)}\right), \iota \in S_{4}$. Here $S_{4}$ is the symmetric group of degree 4 .

So it makes sense for us to come up with a theorem about the congruence classes of quadruples of pairwise distinct points on $\partial \mathbf{H}_{\mathbb{H}}^{n}$ only using geometric invariants. 
Before establishing such a theorem, we suggest a way to evade the embarrassing question caused by $\nu(a)$ and $\sigma(a, b)$. We mention that there exists an interesting Gram matrix given by

$$
\mathbf{m}=\left(\mathbf{p}_{1} \lambda_{1}, \mathbf{p}_{2} \lambda_{2} \lambda_{1}^{-1}, \mathbf{p}_{3} \lambda_{3} \lambda_{1}, \mathbf{p}_{4} \lambda_{4} \lambda_{1}^{-1}\right)
$$

where

$$
\lambda_{2}=\left\langle\mathbf{p}_{2}, \mathbf{p}_{1}\right\rangle^{-1}, \lambda_{3}=\left\langle\mathbf{p}_{3}, \mathbf{p}_{2}\right\rangle^{-1}\left\langle\mathbf{p}_{1}, \mathbf{p}_{2}\right\rangle, \lambda_{4}=\left\langle\mathbf{p}_{4}, \mathbf{p}_{3}\right\rangle^{-1}\left\langle\mathbf{p}_{2}, \mathbf{p}_{3}\right\rangle\left\langle\mathbf{p}_{2}, \mathbf{p}_{1}\right\rangle^{-1}
$$

and

$$
\lambda_{1}=\left|\left\langle\mathbf{p}_{1}, \mathbf{p}_{3} \lambda_{3}\right\rangle\right|^{-1 / 2}=\sqrt{\frac{\left|\left\langle\mathbf{p}_{2}, \mathbf{p}_{3}\right\rangle\right|}{\left|\left\langle\mathbf{p}_{2}, \mathbf{p}_{1}\right\rangle\left\langle\mathbf{p}_{1}, \mathbf{p}_{3}\right\rangle\right|}} .
$$

Computation shows that

$$
G(\mathbf{m})=\left(g_{i j}\right)=\left(\begin{array}{cccc}
0 & 1 & \omega_{3} & \omega_{1} \\
1 & 0 & 1 & \omega_{2} \\
\overline{\omega_{3}} & 1 & 0 & 1 \\
\overline{\omega_{1}} & \overline{\omega_{2}} & 1 & 0
\end{array}\right)
$$

where

$$
\omega_{3}=g_{13}=\frac{\left\langle\mathbf{p}_{1}, \mathbf{p}_{2}, \mathbf{p}_{3}\right\rangle}{\left|\left\langle\mathbf{p}_{1}, \mathbf{p}_{2}, \mathbf{p}_{3}\right\rangle\right|}, \omega_{1}=g_{14}=\frac{\left\langle\mathbf{p}_{2}, \mathbf{p}_{1}\right\rangle \mathbb{X}_{2}(\mathbf{p})\left\langle\mathbf{p}_{1}, \mathbf{p}_{2}\right\rangle}{\left|\left\langle\mathbf{p}_{1}, \mathbf{p}_{2}\right\rangle\right|^{2}}, \omega_{2}=g_{24}=\omega_{3} \mathbb{X}_{3}(\mathbf{p})
$$

A matrix of the form (72) is called a semi-normalised Gram matrix. We represent such a Hermitian matrix by

$$
V(\mathbf{m})=\left(\omega_{1}, \omega_{2}, \omega_{3}\right) .
$$

Let $G$ and $\tilde{G}$ be two semi-normalised Gram matrix represented by $\left(\omega_{1}, \omega_{2}, \omega_{3}\right)$ and $\left(\tilde{\omega}_{1}, \tilde{\omega}_{2}, \tilde{\omega}_{3}\right)$, respectively. By Proposition 5.4 (i), $\tilde{G}$ and $G$ are equivalent if and only if there exists a matrix

$$
D=\mu I_{4}, \mu \in \mathbb{H},|\mu|=1
$$

such that

$$
\tilde{G}=D^{*} G D
$$

That is

$$
\left(\tilde{\omega}_{1}, \tilde{\omega}_{2}, \tilde{\omega}_{3}\right)=\bar{\mu}\left(\omega_{1}, \omega_{2}, \omega_{3}\right) \mu=\left(\bar{\mu} \omega_{1} \mu, \bar{\mu} \omega_{2} \mu, \bar{\mu} \omega_{3} \mu\right) .
$$

Based on the above observation, we define an equivalent relation in $\mathbb{C} \times \mathbb{C} \times \mathbb{C}$ by the above equality and denote it by

$$
\left(\tilde{\omega}_{1}, \tilde{\omega}_{2}, \tilde{\omega}_{3}\right) \simeq\left(\omega_{1}, \omega_{2}, \omega_{3}\right) .
$$

Let $M$ be the set of representations $\left(\omega_{1}, \omega_{2}, \omega_{3}\right)$. Then the configuration space $\mathcal{M}(n)$ can be viewed as the quotient of $\mathrm{M}$ under this equivalent relation. That is $\mathcal{M}(n)=M / \simeq$. The description of $\mathrm{M}$ is left as an exercise for the reader.

We revert to the original topic of this subsection. The following theorem provide a criterion on classification of congruence class of two quadruples of pairwise distinct points in $\partial \mathbf{H}_{\mathbb{H}}^{n}$, which somewhat can be viewed as a description of the above equivalent relation by geometric invariants.

Theorem 5.2. Let $\mathfrak{p}=\left(p_{1}, p_{2}, p_{3}, p_{4}\right)$ and $\mathfrak{q}=\left(q_{1}, q_{2}, q_{3}, q_{4}\right)$ be two quadruple of pairwise distinct points in $\partial \mathbf{H}_{\mathbb{H}}^{n}$. Then there exists an isometry $h \in \mathrm{Sp}(n, 1)$ such that $h\left(p_{1}\right)=q_{1}, h\left(p_{2}\right)=q_{2}, h\left(p_{3}\right)=q_{3}, h\left(p_{4}\right)=$ $q_{4}$ if and only if the following conditions hold:

(i) $\mathbb{X}\left(p_{1}, p_{2}, p_{3}, p_{4}\right) \sim \mathbb{X}\left(q_{1}, q_{2}, q_{3}, q_{4}\right), \mathbb{X}\left(p_{1}, p_{4}, p_{3}, p_{2}\right) \sim \mathbb{X}\left(q_{1}, q_{4}, q_{3}, q_{2}\right)$ and $\mathbb{X}\left(p_{2}, p_{4}, p_{3}, p_{1}\right) \sim \mathbb{X}\left(q_{2}, q_{4}, q_{3}, q_{1}\right)$; 
(ii) $\mathbb{A}_{\mathbb{H}}\left(p_{1}, p_{2}, p_{3}\right)=\mathbb{A}_{\mathbb{H}}\left(q_{1}, q_{2}, q_{3}\right), \mathbb{A}_{\mathbb{H}}\left(p_{1}, p_{2}, p_{4}\right)=\mathbb{A}_{\mathbb{H}}\left(q_{1}, q_{2}, q_{4}\right)$ and $\mathbb{A}_{\mathbb{H}}\left(p_{2}, p_{3}, p_{4}\right)=\mathbb{A}_{\mathbb{H}}\left(q_{2}, q_{3}, q_{4}\right)$.

We need the following properties of quaternions to prove Theorem 5.2 . The proof of them is by direct computations.

Proposition 5.5. (i) For any quaternions $a$ and $b$ we have

$$
\Re(a b)=\Re(a) \Re(b)+\Re(\Im(a) \Im(b)) ;
$$

(ii) For $a=a_{1} \mathbf{i}+a_{2} \mathbf{j}+a_{3} \mathbf{k}$ and $b=b_{1} \mathbf{i}+b_{2} \mathbf{j}+b_{3} \mathbf{k}, a_{i}, b_{i} \in \mathbb{R}$, the angle $\phi$ between vectors $\left(a_{1}, a_{2}, a_{3}\right)$ and $\left(b_{1}, b_{2}, b_{3}\right)$ is

$$
\phi=\arccos \left(\frac{\Re(a \bar{b})}{|a b|}\right)=\arccos \left(\frac{-\Re(a b)}{|a b|}\right) .
$$

Proof of Theorem 5.2. The necessity is clear. Since $\operatorname{Sp}(n, 1)$ acts transitively on $\partial \mathbf{H}_{\mathbb{H}}^{n}$ we may assume that $p_{1}=q_{1}=o$ and $p_{2}=q_{2}=\infty$. For sufficiency we need to find an $h \in G_{o, \infty}$ such that

$$
h\left(p_{3}\right)=q_{3}, h\left(p_{4}\right)=q_{4}
$$

under the conditions (i) and (ii). For convenience, we set

$$
p_{3}=\left(r_{1}, \cdots, r_{n}\right), p_{4}=\left(s_{1}, \cdots, s_{n}\right), q_{3}=\left(z_{1}, \cdots, z_{n}\right), q_{4}=\left(w_{1}, \cdots, w_{n}\right) .
$$

The condition $\mathbb{X}\left(o, \infty, p_{3}, p_{4}\right) \sim \mathbb{X}\left(o, \infty, q_{3}, q_{4}\right)$ implies that

$$
\frac{\left|r_{1}\right|}{\left|s_{1}\right|}=\frac{\left|z_{1}\right|}{\left|w_{1}\right|}, \quad \frac{\Re\left(r_{1} \bar{s}_{1}\right)}{\left|r_{1}\right|\left|s_{1}\right|}=\frac{\Re\left(z_{1} \bar{w}_{1}\right)}{\left|z_{1}\right|\left|w_{1}\right|} .
$$

The condition $\mathbb{X}\left(o, p_{4}, p_{3}, \infty\right) \sim \mathbb{X}\left(o, q_{4}, q_{3}, \infty\right)$ implies that

$$
\frac{\left|r_{1}\right|}{\left|\left\langle\hat{\mathbf{p}}_{3}, \hat{\mathbf{p}}_{4}\right\rangle\right|}=\frac{\left|z_{1}\right|}{\left|\left\langle\hat{\mathbf{q}}_{3}, \hat{\mathbf{q}}_{4}\right\rangle\right|}, \frac{\Re\left(r_{1} \overline{\left\langle\hat{\mathbf{p}}_{3}, \hat{\mathbf{p}}_{4}\right\rangle}\right)}{\left|r_{1}\right|\left|\left\langle\hat{\mathbf{p}}_{3}, \hat{\mathbf{p}}_{4}\right\rangle\right|}=\frac{\Re\left(z_{1} \overline{\left\langle\hat{\mathbf{q}}_{3}, \hat{\mathbf{q}}_{4}\right\rangle}\right)}{\left|z_{1}\right|\left|\left\langle\hat{\mathbf{q}}_{3}, \hat{\mathbf{q}}_{4}\right\rangle\right|} .
$$

The condition $\mathbb{X}\left(\infty, p_{4}, p_{3}, o\right) \sim \mathbb{X}\left(\infty, q_{4}, q_{3}, o\right)$ implies that

$$
\frac{\left|s_{1}\right|}{\left|\left\langle\hat{\mathbf{p}}_{3}, \hat{\mathbf{p}}_{4}\right\rangle\right|}=\frac{\left|w_{1}\right|}{\left|\left\langle\hat{\mathbf{q}}_{3}, \hat{\mathbf{q}}_{4}\right\rangle\right|}, \frac{\Re\left(s_{1}\left\langle\hat{\mathbf{p}}_{3}, \hat{\mathbf{p}}_{4}\right\rangle\right)}{\left|s_{1}\right|\left|\left\langle\hat{\mathbf{p}}_{4}, \hat{\mathbf{p}}_{3}\right\rangle\right|}=\frac{\Re\left(w_{1}\left\langle\hat{\mathbf{q}}_{3}, \hat{\mathbf{q}}_{4}\right\rangle\right)}{\left|w_{1}\right|\left|\left\langle\hat{\mathbf{q}}_{4}, \hat{\mathbf{q}}_{3}\right\rangle\right|} .
$$

The conditions $\mathbb{A}_{\mathbb{H}}\left(p_{1}, p_{2}, p_{3}\right)=\mathbb{A}_{\mathbb{H}}\left(q_{1}, q_{2}, q_{3}\right), \mathbb{A}_{\mathbb{H}}\left(p_{1}, p_{2}, p_{4}\right)=\mathbb{A}_{\mathbb{H}}\left(q_{1}, q_{2}, q_{4}\right)$ and $\mathbb{A}_{\mathbb{H}}\left(p_{2}, p_{3}, p_{4}\right)=\mathbb{A}_{\mathbb{H}}\left(q_{2}, q_{3}, q_{4}\right)$ imply that

$$
\frac{\Re\left(r_{1}\right)}{\left|r_{1}\right|}=\frac{\Re\left(z_{1}\right)}{\left|z_{1}\right|}, \frac{\Re\left(s_{1}\right)}{\left|s_{1}\right|}=\frac{\Re\left(w_{1}\right)}{\left|w_{1}\right|}, \frac{\Re\left(\left\langle\hat{\mathbf{p}}_{4}, \hat{\mathbf{p}}_{3}\right\rangle\right)}{\left|\left\langle\hat{\mathbf{p}}_{4}, \hat{\mathbf{p}}_{3}\right\rangle\right|}=\frac{\Re\left(\left\langle\hat{\mathbf{q}}_{4}, \hat{\mathbf{q}}_{3}\right\rangle\right)}{\left|\left\langle\hat{\mathbf{q}}_{4}, \hat{\mathbf{q}}_{3}\right\rangle\right|} .
$$

By Proposition 5.5 and (73)-(76) , we know that the three angles between pairs of the vectors

$$
\Im\left(\frac{r_{1}}{\left|r_{1}\right|}\right), \Im\left(\frac{s_{1}}{\left|s_{1}\right|}\right), \Im\left(\frac{\left\langle\hat{\mathbf{p}}_{3}, \hat{\mathbf{p}}_{4}\right\rangle}{\left|\left\langle\hat{\mathbf{p}}_{3}, \hat{\mathbf{p}}_{4}\right\rangle\right|}\right)
$$

equal the three angles between the corresponding pairs of the vectors

$$
\Im\left(\frac{z_{1}}{\left|z_{1}\right|}\right), \Im\left(\frac{w_{1}}{\left|w_{1}\right|}\right), \Im\left(\frac{\left\langle\hat{\mathbf{q}}_{3}, \hat{\mathbf{q}}_{4}\right\rangle}{\left|\left\langle\hat{\mathbf{q}}_{3}, \hat{\mathbf{q}}_{4}\right\rangle\right|}\right)
$$

respectively. 
Let

$$
\kappa=\frac{\left|r_{1}\right|}{\left|z_{1}\right|}=\frac{\left|s_{1}\right|}{\left|w_{1}\right|}=\frac{\left|\left\langle\hat{\mathbf{p}}_{3}, \hat{\mathbf{p}}_{4}\right\rangle\right|}{\left|\left\langle\hat{\mathbf{q}}_{3}, \hat{\mathbf{q}}_{4}\right\rangle\right|}
$$

Due to the action of $\mathrm{Sp}(1)$ by conjugation in $\mathbb{H}$ coincides with the action of $\mathrm{SO}(3)$, therefore there exists a $\mu \in \operatorname{Sp}(1)$ such that

$$
r_{1}=\kappa \bar{\mu} z_{1} \mu, \quad s_{1}=\kappa \bar{\mu} w_{1} \mu, \quad\left\langle\hat{\mathbf{p}}_{3}, \hat{\mathbf{p}}_{4}\right\rangle=\kappa \bar{\mu}\left\langle\hat{\mathbf{q}}_{3}, \hat{\mathbf{q}}_{4}\right\rangle \mu .
$$

Noting that

$$
\left\langle\hat{\mathbf{p}}_{3}, \hat{\mathbf{p}}_{4}\right\rangle=r_{1}+\bar{s}_{1}+\sum_{i=2}^{n} \bar{s}_{i} r_{i},\left\langle\hat{\mathbf{q}}_{3}, \hat{\mathbf{q}}_{4}\right\rangle=z_{1}+\bar{w}_{1}+\sum_{i=2}^{n} \bar{w}_{i} z_{i}
$$

we have that

$$
\sum_{i=2}^{n} \bar{s}_{i} r_{i}=\kappa \bar{\mu}\left(\sum_{i=2}^{n} \bar{w}_{i} z_{i}\right) \mu
$$

Since $p_{3}, p_{4}$ and $q_{3}, q_{4}$ are on $\partial \mathbf{H}_{\mathbb{H}}^{n}$, we have that

$$
\frac{\sum_{i=2}^{n}\left|r_{i}\right|^{2}}{\sum_{i=2}^{n}\left|z_{i}\right|^{2}}=\frac{\sum_{i=2}^{n}\left|s_{i}\right|^{2}}{\sum_{i=2}^{n}\left|w_{i}\right|^{2}}=\kappa
$$

and so that we can find an $A \in \operatorname{Sp}(n-1)$ such that

$$
A\left(r_{2}, \cdots, r_{n}\right)^{T}=\sqrt{\kappa}\left(z_{2}, \cdots, z_{n}\right)^{T} \mu, A\left(s_{2}, \cdots, s_{n}\right)^{T}=\sqrt{\kappa}\left(w_{2}, \cdots, w_{n}\right)^{T} \mu .
$$

Hence $h=\operatorname{diag}\left(\frac{\mu}{\sqrt{\kappa}}, A, \sqrt{\kappa} \mu\right)$ is the desired isometry.

Acknowledgements This work was supported by National Natural Science Foundation of China and Educational Commission of Guangdong Province. The authors would like to thank Prof. John R. Parker and the referee for their useful suggestions, which totally reshaped and enhanced this paper.

\section{References}

[1] Apanasov, B. N., Kim, I.: Cartan angular invariant and deformations of rank 1 symmetric spaces. Sbornik: Mathematics 198(2), 147-169 (2007)

[2] Beardon, A. F.: The geometry of discrete groups, Berlin, New York: Spring-Verlag, (1983)

[3] Bisi, C., Gentili, G.: Möbius transformations and the Poincare distance in the quaternionic setting. Indiana Univ. Math. J. 58, 2729-2764 (2009)

[4] Brehm, U.: The shape invariant of triangles and trigonometry in two-point homogeneous spaces. Geometriae Dedicata. 33, 59-76 (1990)

[5] Cao, C., Waterman, P. L.: Conjugacy invariants of Möbius groups, Quasiconformal Mappings and Analysis. Springer, 109-139 (1998)

[6] Cao, W. S., Parker, J. R.: Jørgensen's inequalities and collars in n-dimensional quaternionic hyperbolic space. Quarterly J. Math. 62, 523-543 (2011)

[7] Cartan, E.: Sur le groupe de la géométrie hypersphérique. Comment. Math. Helv. 4, 158-171 (1932) 
[8] Chen, S.S., Greenberg, L.: Hyperbolic spaces, Contributions to analysis. Academic Press, New York. 49-87 (1974)

[9] Cunha, H., Gusevskii, N.: On the moduli space of quadruples of points in the boundary of complex hyperbolic space. Transform. Groups 15(2), 261-283 (2010)

[10] Cunha, H., Gusevskii, N.: The moduli space of points in the boundary of complex hyperbolic space. J. Geom. Anal. 22, 1-11 (2012)

[11] Falbel, E., Platis, I. D.: The PU $(2,1)$ confguration space of four points in $S^{3}$ and the cross-ratio variety. Math. Ann. 340(4), 935-962 (2008)

[12] Falbel, E.: A spherical CR structure on the complement of the fgure eight knot with discrete holonomy. J. Diferential Geom. 79(1), 69-110 (2008)

[13] Goldman, W. M.: Complex hyperbolic geometry. In: Oxford Mathematical Monographs. Oxford Science Publications. The Clarendon Press, Oxford University Press, New York (1999)

[14] Grossi, C.: PhD Thesis, Universidade Estadual de Campinas, (2006)

[15] Kim, I., Parker, J. R.: Geometry of quaternionic hyperbolic manifolds. Math. Proc. Cambridge Philos. Soc. 135, 291-320 (2003)

[16] Korányi, A., Reimann, H. M.: The complex cross-ratio on the Heisenberg group. Enseign. Math. 33, 291-300 (1987)

[17] Parker, J. R.: Notes on complex hyperbolic geometry. (2010).

[18] Parker, J. R., Platis, I. D.: Complex hyperbolic Fenchel-Nielsen coordinates. Topology 47(2), 101-135 (2008)

[19] Parker, J. R., Platis, I. D.: Global geometrical coordinates on Falbel's cross-ratio variety. Canad. Math. Bull. 52, 285-294 (2009)

[20] Platis, I. D.: Cross-ratios and the Ptolemaean inequality in boundaries of symmetric spaces of rank 1. Geometriae Dedicata. 169, 187-208 (2014) 